CUAD. CONTAB. / BOGOTÁ, COLOMBIA, 17 (44): 545-573 / JULIO-DICIEMBRE 2016 / 545

\title{
El uso de la estadística en la ejecución de trabajos de auditoría financiera*
}

doi:10.11144/Javeriana.cc17-44.ueet

\section{Fabio Enrique Gómez-Meneses}

Contador público. Especialista en revisoría fiscal y auditoría externa, Universidad Autónoma de Bucaramanga.

Máster en administración electrónica de empresas y máster en contabilidad y finanzas, Universidad de Zaragoza, España. Doctor en contabilidad y finanzas, la Universidad de Zaragoza, España. Profesor de tiempo completo, Facultad de Ciencias Económicas, Administrativas y Contables, Universidad Autónoma de Bucaramanga, UAB.

Correo electrónico: fgomezm@unab.edu.co

* Artículo derivado del proyecto de investigación Análisis de la estadística y la econometría en la aplicación de trabajos de auditoría financiera. Proyecto realizado durante 2014 y financiado por la Universidad Autónoma de Bucaramanga. Código del proyecto: I-34100. 
Resumen Cuando se realizan auditorías en las organizaciones empresariales es probable que se manejen altos volúmenes de información financiera y contable, lo cual genera la necesidad de utilizar ciertas técnicas estadísticas y econométricas que faciliten la recolección y análisis de los datos, para obtener evidencia suficiente que dé seguridad razonable respecto a que la información analizada refleja la realidad económica de una determinada compañía. Teniendo en cuenta lo anterior, en el presente documento se profundizará de manera teórica y práctica en el uso de la estadística en la ejecución de trabajos de auditoría financiera, como herramienta de gran significatividad para que los trabajos de este tipo tengan la mayor calidad posible. Para ello, el documento se estructura en tres partes, la introducción y marco teórico, el diseño metodológico y los ejemplos descriptivos y, finalmente, las conclusiones, las cuales están enfocadas en la contribución de este documento a la mejora de los procesos de formación de los contadores públicos en lo que se refiere al desempeño profesional en un trabajo de auditoría financiera.

Palabras clave Auditoría financiera; estadística; econometría; contador público; auditor

\section{Código JEL M42}

\section{Use of Statistics in the Performance of Financial Audit Works}

\footnotetext{
Abstract When conducting audits in business organizations, high volumes of financial and accounting information are likely to be handled. This, in turn, leads to the need to use certain statistical and econometric techniques to facilitate the collection and analysis of data as to obtain sufficient evidence that provides reasonable assurance that the information analyzed reflects the economic reality of a particular company. Taking the above into account, in this document we will deepen in a theoretical and practical way into the use of statistics in the performance of financial audit work, as a very significant tool to ensure works of this type have the highest possible quality. To this end, the doc-
}

ument is structured in three parts, introduction and theoretical framework, methodological design and descriptive examples and, finally, the conclusions, which are focused on the contribution of this document to the improvement of the training processes of public accountants in terms of professional performance in a financial audit work.

Keywords Financial audit; statistics; econometrics; public accountant; auditor

\section{O uso da estatística na execução de trabalhos de auditoria financeira}

Resumo Quando auditorias nas organizações empresariais são feitas, é provável manusear altos volumes de informação financeira e contábil, o qual gera a necessidade de utilizar certas técnicas estatísticas e econométricas facilitando a coleta e análise de dados para obter evidencia suficiente que forneça segurança razoável respeito de que a informação analisada reflete a realidade económica de uma determinada companhia. Levando em conta o dito, no presente documento aprofundar-se-á de maneira teórica e prática no uso da estatística na execução de trabalhos de auditoria financeira, como ferramenta de grande significância para que os trabalhos deste tipo tiver a maior qualidade possível. Para isso, o documento é estruturado em três partes, introdução e quadro teórico, design metodológico e exemplos descritivos e, no fim, as conclusões, as quais são focadas nas contribuições deste documento para o melhoramento dos processos de formação dos contadores públicos no que diz respeito do desempenho profissional em um trabalho de auditoria financeira.

Palavras-chave Auditoria financeira; estatística; econometria; contador público; auditor

\section{Introducción}

Las organizaciones empresariales llevan a cabo y procesan una enorme cantidad de transacciones 
EL USO DE LA ESTAdíStICA EN LA EJECUCIÓN DE TRABAJOS DE AUDITORÍA FINANCIERA / F. GóMEZ / 547

contables derivadas de las diferentes actividades que desarrollan en cumplimiento de sus objetivos. Todas estas actividades y transacciones deben ser revisadas y evaluadas, de tal forma que aquellos usuarios de la información que estén interesados en las cifras, balances y cuentas que presentan las compañías, puedan tener el suficiente grado de confiabilidad en la información que se reporta.

Esta labor de revisión y evaluación se puede llevar a cabo mediante un proceso de auditoría y, más específicamente, de una auditoría financiera, proceso que estará en cabeza de un contador público o a cargo de una firma de contadores. La ejecución de un trabajo de auditoría financiera, junto con otras clases de auditoría (cumplimiento, sistemas, tributaria, gestión, etc.), permitirá evaluar el cumplimiento de las empresas en tres aspectos trascendentales para su correcta operación: la razonabilidad de los estados financieros, el cumplimiento de las normas aplicables y la uniformidad en la aplicación de tales normas.

Ahora bien, en los procesos de auditoría, es probable que se tenga que trabajar con grandes volúmenes de información. Esta situación puede generar dificultades, si no se utilizan las herramientas necesarias para su manejo, lo cual disminuye la confiabilidad del trabajo realizado por el auditor.

Para dar respuesta a esta problemática, podemos recurrir al uso de la estadística y de la econometría, herramientas que facilitarán significativamente la recolección, tratamiento y análisis de los datos, de tal forma que el auditor pueda brindar una opinión con base en evidencias técnicamente recabadas, lo que mejora la calidad de su trabajo, para dar un mayor grado de seguridad razonable respecto a si la información contable analizada refleja la realidad económica de una determinada organización.

De acuerdo a lo anterior, y siendo conscientes de las debilidades que estudios e investigaciones previas han encontrado en la formación del contador público en Colombia y, en general, en países de tradición latina, como los trabajos de María Antonia García-Benau y Antonio Vico-Martínez (2003), Fabio Gómez (2014), Gabriel Rueda-Delgado, Jorge Emiro PinzónPinto y Ruth Alejandra Patiño-Jacinto (2013) y Gerardo Santos y Ruth Alejandra Patiño-Jacinto (2009), es necesario profundizar en este aspecto, es decir, en el uso de herramientas estadísticas en el desarrollo de una auditoría financiera, ya que su no aplicación implicaría una falta de rigurosidad en los trabajos de auditoría efectuados y, en consecuencia, un menor grado de confiabilidad en los resultados obtenidos.

En este sentido, en el presente documento se llevará a cabo un recorrido teórico para analizar, de forma conceptual, de qué manera la estadística puede ser usada en la ejecución de un trabajo de auditoría financiera. Una vez realizada esta revisión teórica, se presentarán y describirán una serie de ejemplos que le permitan al lector la mayor comprensión posible. Finalmente, con base en las temáticas desarrolladas en el presente documento, se formularán conclusiones y recomendaciones en cuanto a la aplicación de la estadística por parte de los contadores públicos.

\section{Marco teórico}

La auditoría es un área de la profesión contable que ha evolucionado a través de los siglos. Con 
el transcurrir de los años, sus objetivos se han movido desde la detección de fraudes o malversaciones, pasando por la revisión de sistemas de control interno, hasta el análisis y evaluación de los procedimientos y políticas de una entidad (Porter \& Burton, 1980).

A la par con esta evolución, en cuanto a sus objetivos se refiere, poco a poco también se ha modificado la manera de llevar a cabo esta importante labor. Hoy se dispone de la posibilidad de utilizar las tecnologías de la información y la comunicación - TIC - en el desarrollo de un trabajo de auditoría, con el ánimo de disminuir los costos y tiempos en las diferentes actividades realizadas; se obtienen así ventajas competitivas y un mejor manejo de la información (Barua, Kriebel \& Mukhopadhyay, 1995; Luo, Cook, Joseph \& Ganapathy, 2000; Valderrama-Prieto, 2002). Igualmente, en la ejecución de trabajos de auditoría, se comienza a utilizar una amplia diversidad de técnicas, herramientas y metodologías estadísticas y econométricas que facilitan y aumentan la calidad del trabajo realizado por el auditor, técnicas que han venido siendo utilizadas en la economía y en la administración y dirección de empresas (Miró i Martínez, Debón \& Crespo, 2006; Rubio-Andrada, 2005).

Ahora bien, en estas tres cuestiones (utilización de TIC, estadística y econometría), los contadores públicos de algunos países, sobre todo de tradición latina, al parecer no están suficientemente preparados o capacitados, como bien describen trabajos como los de Ángelo Benvenuto-Vera (2004), María Antonia García-Benau y Antonio Vico-Martínez (2003) y Hugo Gub- ba, Jorge Gutfraind, Rubén Rodríguez y Ricardo Villarmarzo (2001), o la investigación de Fabio Gómez (2014). En este último trabajo, se analizaron una serie de factores relacionados con el ejercicio de la auditoría por parte de los profesionales contables en Colombia, entre los cuales figura la estadística.

Los resultados de este estudio son abiertamente negativos; es decir, la estadística es muy poco usada en la realización de trabajos de auditoría, por parte de los contadores/auditores encuestados, lo cual expone a las organizaciones empresariales a un sinnúmero de riesgos que pueden afectar su estabilidad en el tiempo.

Es de esperar que esta debilidad en particular (el no uso de la estadística) tenga un impacto directo en las competencias del auditor para la detección de fraudes o de situaciones indeseables en las empresas, situación que puede constituirse como caldo de cultivo para eventos de corrupción empresarial que terminan minando la confianza del público en general sobre el ejercicio profesional de la auditoría, tal como exponen María Antonia García-Benau y Antonio Vico-Martínez (2003) y Fabio Gómez (2014).

Ante este panorama, y como bien lo indica Enrique Bonsón-Ponte (2000), se plantea un nuevo desafío para el auditor, que para el contexto de este trabajo, se centrará específicamente en el uso de la estadística como herramienta fundamental para el buen ejercicio de la auditoría. En los siguientes párrafos se conceptualizará acerca de cómo esta herramienta puede ser utilizada para el óptimo desarrollo de un trabajo de auditoría. 


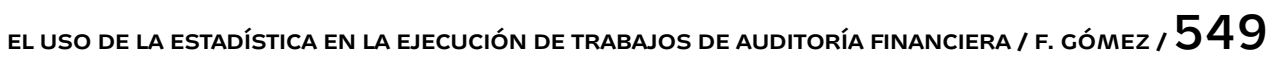

\subsection{Las herramientas estadísticas aplicadas en una auditoría financiera}

El trabajo del auditor implica, en muchas ocasiones, el análisis y revisión de grandes cantidades de datos y cifras, tarea que puede llevarse a cabo con herramientas y metodologías estadísticas que permitan obtener medidas confiables, que soportarán, más razonablemente, las conclusiones y recomendaciones que un auditor realice.

Gracias a la estadística, como se desarrollará más profundamente en los siguientes apartados, el auditor obtendrá importantes beneficios al ejecutar su trabajo; por ejemplo, se podrán administrar los datos de forma más organizada o se podrá obtener un significativo nivel de confianza y fiabilidad en el cálculo de muestras que se utilizarán en las pruebas de auditoría.

Con el uso e interrelación de la estadística se logra una mejor aplicación de la auditoría, en particular, en lo que se refiere a la auditoría financiera, y con la necesaria combinación de esta herramienta y las TIC, el auditor podrá obtener mejores resultados en las evaluaciones que haga, lo que mejorará significativamente la calidad de los conceptos que emita en los respectivos informes de auditoría al final de un proceso de este tipo.

\subsection{Definiciones y conceptos}

Antes de identificar cómo la estadística puede contribuir en el desarrollo de una auditoría financiera, resulta importante definir conceptualmente estos dos términos. Para ello, se parte en primera instancia del concepto de auditoría, que en palabras de Marco Deschamps (2007) es definida como: aquella actividad que consiste en la recopilación y evaluación de datos contables y no contables sobre información cuantificable de un organismo o dependencia, área o actividad para determinar mediante la adecuada aplicación de los criterios establecidos por el auditor, la existencia de irregularidades $y$, en su caso, emitir recomendaciones para corregir desviaciones y verificar que estas se lleven a cabo (p. 6).

Esta definición analiza la auditoría desde un punto de vista global, es decir, no se centra en un área particular de la auditoría (sistemas, financiera, gestión, etc.). Por tal razón, es necesario explorar de forma específica el concepto objeto de estudio en esta investigación, auditoría financiera, para ello nos remitimos a la definición planteada por Guillermo Adolfo Cuéllar-Mejía (2009), que la entiende como "aquel tipo de auditoría que emite un dictamen u opinión profesional en relación con los estados financieros de una unidad económica en una fecha determinada y sobre el resultado de las operaciones y los cambios en la posición financiera" (p. 19).

El segundo concepto a definir es el de la estadística que, según Luz M. Rivera (2006), se entiende como:

una colección de métodos para planificar y realizar experimentos, obtener datos y luego analizar, interpretar y formular una conclusión basada en esos datos. Es la ciencia encargada de recopilar, organizar, analizar e interpretar información numérica o cualitativa 
de manera que pueda llevar a conclusiones válidas (p. 1).

A la vista de estas definiciones, la estadística puede resultar muy útil en la ejecución de un trabajo de auditoría financiera; de hecho, en el contexto actual en el que se desarrolla la economía, el uso de la estadística se convierte en una necesidad y obligación, si el auditor quiere ofrecer un trabajo de calidad. Como bien se plantea en la definición de Luz M. Rivera (2006), la estadística obtiene, analiza e interpreta datos, a partir de los cuales se genera una serie de conclusiones. Precisamente, esta es una de las tareas que tiene que llevar a cabo el auditor: obtener, analizar e interpretar datos financieros y contables para dar una opinión o conclusión sobre la materia objeto de estudio.

Siendo esto así, no hay ninguna duda de que en muchas ocasiones es factible y probablemente necesario, usar la estadística en un trabajo de auditoría financiera. Ahora bien, el cuestionamiento lógico que el auditor debe hacerse es: ¿de qué manera y en qué actividad en particular de su trabajo de auditoría, involucrará el uso de la estadística? Esta importante pregunta será resuelta en el siguiente apartado.

\subsection{Aplicaciones de la estadística en la auditoría financiera}

En los siguientes párrafos se abordarán conceptualmente tres posibles aplicaciones de la estadística en un trabajo de auditoría financiera: el cálculo de muestras; la agrupación y presentación de los datos; y la probabilidad de ocurrencia de situaciones de riesgo. Posteriormente, se mostrarán algunos ejemplos relacionados.

\section{- El cálculo de muestras}

Hay muchas formas de aplicar la estadística en un trabajo de auditoría. Se va a iniciar con la que tal vez los auditores consideran como la más relevante: aquella que hace referencia al cálculo de muestras cuando se realizan pruebas de auditoría. El tema es tan relevante que múltiples autores han desarrollado trabajos al respecto, como Alvin A. Arens y James Loebbecke (1981), Roberto Escuder-Vallés y Salvador Méndez-Martínez (2002), Ghanzov Jokovich (2013), Salvador Méndez-Martínez, Roberto Escuder-Vallés y Cecilio Mar-Molinero (2001) y Kenneth Stringer (1963).

En los cuatro primeros trabajos reseñados, los autores analizan los diferentes modelos de muestreo existentes y cómo se aplican estos al desarrollo de auditorías en las empresas, teniendo en cuenta el juicio de auditor $y$, en cuanto al quinto trabajo relacionado, este se centra en investigar mediante simulaciones, si la técnica de remuestreo bootstrap es adecuada o no para el objetivo de una auditoría.

Inicialmente, hay que indicar, de acuerdo a lo planteado por Richard I. Levin y David S. Rubin (2010), que se debe recurrir al muestreo cuando no es posible contar o medir todos los elementos de la población. Para la temática que nos convoca (auditoría financiera), supongamos que el auditor debe dar una opinión sobre la razonabilidad y veracidad de los saldos de cartera existentes en una empresa multinacional y también supongamos que estamos hablando de que esta organización tiene miles de clientes alrededor del mundo. Resultaría impracticable hacer esta revisión cliente por cliente, por lo tanto, es este el momento en el 


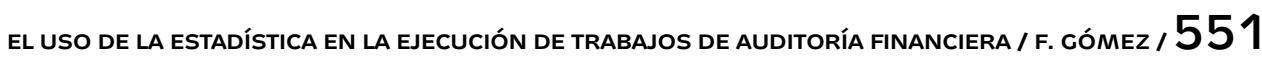

cual podemos sacar partido de la estadística, mediante la selección de una muestra que resulte representativa del total de la población de clientes.

Para determinar esta muestra, se podría recurrir a alguna de las dos clases de muestreo existentes: muestreo no aleatorio y muestreo aleatorio. El muestreo no aleatorio consiste simplemente en seleccionar la muestra de acuerdo a la experiencia y juicio personal de quien vaya a ejecutar el trabajo, es decir, para nuestro caso, el auditor, que gracias a su experiencia en la ejecución de auditorías financieras y a su conocimiento en las categorías de clientes existentes en la multinacional, puede decidir por ejemplo, seleccionar solamente los clientes de las 5 capitales más grandes donde la organización comercializa sus productos y servicios. Este muestreo tiene importantes desventajas, entre ellas podemos mencionar que no resulta muy representativo y no es posible evaluar su validez. Por estas razones, en particular por su simplicidad, no se tendrá en cuenta en el desarrollo de este documento.

En cuanto al muestreo aleatorio, con él se pueden llevar a cabo, de forma rigurosa, todas las mediciones y análisis estadísticos respectivos. Esta es la categoría de muestreo que deberían utilizar los auditores, sin que esto quiera decir que el muestro no aleatorio no resulte válido en ciertas situaciones que el auditor considere pertinentes con base en su experiencia y conocimiento.

El muestreo aleatorio se puede llevar a cabo de cuatro maneras o formas: muestreo aleatorio simple, muestreo sistemático, muestreo estratificado y muestreo de racimo. El primero de estos métodos parte de la premisa de que en una determinada población, cada elemento debe tener la misma posibilidad que los demás de ser tenida en cuenta para la muestra. En el muestreo sistemático, al determinar una muestra, sus elementos se eligen mediante intervalos uniformes, es decir, se mantienen iguales. Estos intervalos pueden ser diseñados en función del tiempo, orden o espacio (Deschamps, 2007; Levin \& Rubin, 2010).

El muestreo aleatorio estratificado consiste simplemente en separar una determinada población en grupos que sean lo más homogéneos posible; estos grupos toman el nombre de estratos, de ahí el nombre de muestreo estratificado. Finalmente, en el muestreo de racimo, que es similar al método anterior, se separa la población en grupos o racimos, para luego seleccionar en cada uno de estos racimos una muestra aleatoria sobre la cuales se efectuarán las consultas respectivas. La diferencia esencial entre estos dos métodos que parecen similares, es que en el muestreo estratificado hay diferencias importantes entre un grupo y otro, pero no dentro de cada grupo, mientras que en el muestreo de racimo, no hay variaciones significativas entre un grupo y otro, pero sí las hay dentro de cada grupo.

Cada uno de estos métodos tiene sus ventajas y desventajas; por ejemplo, en el muestreo estratificado, si este está bien realizado, las muestras evidenciarán de forma más específica las características de la población. Para el caso del muestro sistemático, hay riesgos respecto a la falta de representatividad de la muestra, aunque esto puede verse compensando por su facilidad y menor costo (Deschamps, 2007; Levin 
\& Rubin, 2010). La decisión de utilizar uno u otro método dependerá de la población y sus elementos, de cómo está organizada tal población y, lógicamente, del juicio, conocimientos y capacidad que tenga el auditor.

El objeto del presente documento no es brindar un curso de estadística a profundidad a los auditores, pero sí indicar la importancia de que el profesional contable tenga tales conocimientos estadísticos que mejoren su juicio y capacidad, para lo cual el auditor tiene la responsabilidad de subsanar estas debilidades mediante el estudio y consulta de textos sobre el tema. De los muchos existentes, se recomiendan el de Damodar N. Gujarati (1992) y, en particular, el de Richard I. Levin y David S. Rubin (2010). En este último libro se aborda la estadística, desde la perspectiva de los negocios, lo cual resulta supremamente útil para un fácil entendimiento, pero mejor aún, para una decidida aplicación en el ámbito profesional que nos compete.

\section{- La agrupación y presentación de los datos}

Una segunda área en la cual podemos aplicar la estadística en la ejecución de trabajos de auditoría es la que se refiere a la agrupación y presentación de los datos. Si los datos están correctamente tabulados e incluso, graficados, estos se convertirán en información, con la cual se podrá llegar a conclusiones razonables y confiables que faciliten el proceso de toma de decisiones en las organizaciones y del propio auditor.

Esta agrupación y presentación de los datos — “ordenamiento de datos"-involucra sencillos procesos estadísticos como la organización de valores en orden ascendente o descendente, la identificación de valores mínimos y máximos, la división de los datos en secciones o grupos, la repetición de datos y la distancia existente entre los valores que conforman una sección en particular (Deschamps, 2007; Levin \& Rubin, 2010).

Ahora bien, si queremos profundizar un poco más en nuestra ordenación y presentación de datos, podemos recurrir a otros procedimientos estadísticos que no revisten gran dificultad, como las distribuciones de frecuencias, las medidas de tendencia central, como la media, mediana y moda, y las medidas de dispersión, como la desviación promedio y el coeficiente de variación.

En términos sencillos, una distribución de frecuencias sirve para agrupar datos numéricos y detalla cada uno de los valores diferentes que conforman el total de datos, al igual que el número de veces que aparece cada dato, que es lo que llamamos frecuencia. De otra parte, si hablamos de las medidas de tendencia central, podemos identificarlas como aquellos puntos o elementos de referencia que nos pueden dar una indicación o conclusión general y aproximada sobre una muestra de datos o sobre un dato en particular. Finalmente, las medidas de dispersión indican la confiabilidad de las medidas de tendencia central y la dispersión de los datos, es decir, se evidencia qué tan representativa es la posición central determinada en los datos analizados (Deschamps, 2007; Levin \& Rubin, 2010).

\section{- Probabilidad de ocurrencia de situaciones de riesgo}

Por último, una tercera forma en la cual podemos aplicar la estadística en trabajos de audito- 


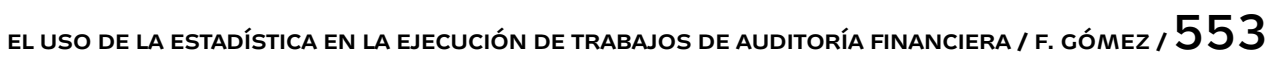

ría financiera es el proceso de identificación y valoración de riesgos en las empresas, riesgos que pueden generar pérdidas económicas a las organizaciones. El auditor tiene la tarea fundamental de identificar los riesgos a los cuales está expuesta una compañía en un área en particular; una vez identificados tales riesgos, estos deberán ser estimados y cuantificados, para lo cual el auditor tomará en consideración dos aspectos: el costo o valor de las pérdidas en que incurriría la organización cada vez que el riesgo se materialice y su probabilidad de ocurrencia.

En este último factor, el auditor podrá soportarse en la estadística, específicamente en la teoría de la probabilidad. El concepto o definición de probabilidad es bastante simple: se trata de la posibilidad de que algo suceda; por ejemplo, la posibilidad de que la organización gane o pierda un litigio; la posibilidad de que un cliente nos pague o no; la probabilidad de que se produzcan alteraciones y manipulaciones de información o no en la empresa.

Lógicamente, aplicar la teoría de la probabilidad nos permitirá tomar decisiones más estructuradas y lógicas como auditores al momento de evaluar riesgos. Sin lugar a dudas, esto repercutirá en la calidad del resultado final que arroje nuestro trabajo de auditoría, con los beneficios que tal situación supone para la organización empresarial que se esté auditando.

Hay muchas más aplicaciones de la estadística en un trabajo de auditoría financiera, esto dependerá del tipo de trabajo que se efectúe y de las necesidades del mismo. De manera ilustrativa, en los párrafos precedentes se presentaron tres (3) de ellas que pueden ser muy relevantes para un auditor y que, de ser apli- cadas, contribuirán sensiblemente al mejoramiento de los trabajos de auditoría financiera en las empresas.

\subsection{Otras herramientas a considerar}

Si bien este trabajo se centra en cómo la estadística puede ser de gran utilidad en la ejecución de trabajos de auditoría financiera, también resulta importante hacer una breve alusión al uso de la econometría.

De acuerdo a lo planteado por Damodar N. Gujarati (1997), la econometría consiste en "llevar a cabo mediciones económicas, teniendo en cuenta la teoría económica, la economía matemática, la estadística económica y la estadística matemática, todo ello con el ánimo de lograr entender y predecir, por ejemplo, el comportamiento de los negocios y del mercado" (p. 1).

Resulta claro - como describen algunos trabajos sobre el tema, como el de Víctor Raúl López-Ruiz y Domingo Nevado-Peña (1999)— que la aplicación de la econometría facilita y agiliza la ejecución de ciertas etapas en el proceso de auditoría, pues optimiza tiempos, reduce costos y mejora la calidad del trabajo e informe final presentado. De forma más específica se podría plantear que mediante el diseño y aplicación de modelos econométricos, se podrán determinar y representar las cuentas que revistan mayor interés para un auditor y en las cuales se pueden presentar inconsistencias.

Igualmente, mediante la econometría se pueden identificar variaciones y la evolución en partidas y cuentas que puedan tener una gran significatividad. Lógicamente, tener esta información sobre algunas partidas que revistan 
materialidad, repercute en la decisión del auditor al momento de determinar la cantidad y extensión de las pruebas de auditoría efectuadas, tanto sustantivas como de cumplimiento, es decir, estas pruebas estarán centradas de forma específica en lo que realmente la empresa auditada necesita que se evalúe y no estarán dispersas en revisiones superfluas que probablemente no aporten nada en el desarrollo del examen de auditoría.

Finalmente, gracias a la aplicación de modelos econométricos, se pueden hacer evaluaciones de control interno de forma más rápida y certera, para mejorar significativamente la fiabilidad y razonabilidad y disminuir la probabilidad de error en los resultados obtenidos ${ }^{1}$.

\section{Diseño metodológico}

El trabajo de investigación en cuestión es descriptivo, ya que en él se hace una aproximación directa a un problema existente en la profesión contable al identificar las causas que lo generan (Hernández-Sampieri, Fernández \& Baptista, 2010); es diacrónico, porque se trata de una situación que se está presentando a lo largo de un período extenso, lo cual implica que se puedan presentar cambios en el fenómeno estudiado; y es cualitativo, porque se busca estudiar y describir un suceso o problema con información principalmente cualitativa, todo ello con el objetivo de mejorar el ejercicio profesional

1 Si se requiere profundizar sobre la econometría, se recomienda consultar los textos de Aurora Alonso-Antón, Francisco Javier Fernández-Macho e Inmaculada Gallastegui-Zulaica (2005), Arthur S. Goldberger (1991), Damodar N. Gujarati (1997), Paul Samuelson, Tjalling Koopmans y Richard Stone (1954) y Jeffrey M. Wooldridge (2007). de los contadores, con una serie de ejemplos que permitan analizar y describir lo que se plantea conceptualmente.

Para la ejecución de esta investigación, el primer paso supuso partir de una revisión bibliográfica, soportada en libros y artículos publicados en revistas y bases de datos electrónicas que abordan esta temática y que permitió la identificación de los principales métodos y herramientas estadísticas disponibles y aplicables en el desarrollo de un trabajo de auditoría financiera.

A lo largo de la ejecución de esta primera parte de la investigación, que corresponde a la identificación de las bases teóricas y conceptuales, se contó con el soporte de dos (2) estudiantes del programa de contaduría pública de la institución universitaria que financió el proyecto de investigación; vincular a estudiantes de pregrado en la formulación y ejecución de proyectos de investigación contribuye a su formación en investigación.

Agotada esta primera parte del estudio, se llevó a cabo el respectivo análisis de los diferentes métodos y herramientas estadísticas, de manera que se logre su adecuada comprensión, para permitir el desarrollo de ejemplos que expliquen algunos de los métodos identificados y analizados.

Estos casos desarrollados con los programas Excel y SPSS le permitirán al contador/auditor contextualizar en su práctica profesional, una serie de herramientas estadísticas que mejorarán la calidad del trabajo de auditoría financiera que efectúe, con las consecuencias positivas que esto supone para las organizaciones empresariales.

La ejecución de este trabajo tiene un significativo valor metodológico, ya que se pretende facilitar el entendimiento y aplicación 
EL USO dE LA ESTAdíStICA EN LA EJECUCIÓN DE TRABAJOS DE AUDITORÍA FINANCIERA / F. GóMEZ / 55

de métodos y herramientas estadísticas en una auditoría financiera, mediante la presentación de ejemplos que permitan su adecuada descripción y, lo más importante, su utilización por parte de la comunidad nacional de contadores y auditores, todo ello, en beneficio de la organización evaluada.

\section{Ejemplos de estadística aplicados en la auditoría financiera}

En el primer ejemplo que se presenta, se van a efectuar una serie de procedimientos estadísticos sobre las cifras financieras de una supuesta base de datos de 250 empresas clientes, de una ficticia firma de auditoría internacional; en particular, se recurrirá a la agrupación y ordenamiento de los datos, para posteriormente revisar el cálculo de muestras estadísticas, en el segundo de los ejemplos relacionados.

En cuanto al tercer ejemplo, se describirán las acciones necesarias para determinar la probabilidad de subsistencia o quiebra de las organizaciones analizadas. Este análisis se soporta en la teoría de la probabilidad.

Respecto al cuarto y último ejemplo, se tratará de establecer si es posible determinar un perfil financiero específico, tanto para las empresas que obtienen dictámenes positivos de auditoría como para aquellas que obtienen dictámenes negativos en sus evaluaciones.

\section{- Ejemplo I}

Para este este primer ejemplo, supongamos que se va a trabajar con la información financiera de 250 empresas clientes de una ficticia firma de auditoría internacional, específicamente con las cifras de ventas, utilidad neta, activos totales y patrimonio neto de estas compañías para 2012. Los datos en cuestión pueden ser consultados en el Anexo 1. Inicialmente, se ordenarán estos datos; para ello, se deberán ejecutar de forma específica los siguientes procedimientos:

- Organización en orden ascendente o descendente de las cifras

- Identificación de los valores mínimos y máximos

- Clasificación de los datos en secciones o grupos

- Cálculo de distribución de frecuencias

- Cálculo de medidas de tendencia central (media, mediana y moda)

- Cálculo de medidas de dispersión (desviación estándar y varianza)

Partiendo de los datos cuantitativos señalados y de su ordenamiento inicial, bien sea este en forma ascendente o descendente, en la tabla 1 se presentan y describen algunos de los aspectos relacionados con la organización de los datos.

Las medidas estadísticas calculadas y presentadas en la tabla 1 le permiten a quien esté analizando los datos formarse una idea sobre las cifras que están sujetas a estudio o auditoría. Por ejemplo, con estos resultados iniciales se pueden observar los valores promedio en cada una de las cuatro (4) variables financieras; esta información puede serle útil al auditor para conocer el comportamiento general de las organizaciones. 


\begin{tabular}{lrrrrrrr}
\hline \multicolumn{1}{c}{ Variable } & $\begin{array}{c}\text { Valor } \\
\text { mínimo }\end{array}$ & $\begin{array}{c}\text { Valor } \\
\text { máximo }\end{array}$ & Media & Mediana & Moda & Varianza & $\begin{array}{c}\text { Desviación } \\
\text { estándar }\end{array}$ \\
\hline Ventas 2012 & $1.549,7$ & $137.694,9$ & $6.694,9$ & $2.994,2$ & $2.247,6$ & $181.656 .458,0$ & $13.477,9$ \\
$\begin{array}{l}\text { Utilidad neta } \\
\mathbf{2 0 1 2}\end{array}$ & 2,6 & $10.365,8$ & 553,9 & 226,6 & 25,9 & $1.376 .947,5$ & $1.173,4$ \\
$\begin{array}{l}\text { Activo total } \\
\mathbf{2 0 1 2}\end{array}$ & 277,0 & $331.644,9$ & $9.156,2$ & $4.395,1$ & & & \\
$\begin{array}{l}\text { Patrimonio neto } \\
\mathbf{2 0 1 2}\end{array}$ & 65,7 & $167.888,3$ & $3.862,9$ & $1.749,1$ & $1.014,6$ & $186.492 .315,0$ & $13.656,2$ \\
\hline
\end{tabular}

Tabla 1

Estadísticos descriptivos de la base de datos analizada Fuente: elaboración propia

Si el auditor quiere profundizar un poco más en este primer análisis, puede clasificar los datos en secciones o construir una serie de grupos con base en el país al cual pertenecen las diferentes empresas o en el sector económico en el cual se desarrollan estas compañías, para luego volver a aplicar las medidas estadísticas, pero esta vez, para cada uno de los grupos construidos, con lo cual podría conocer, por ejemplo, el desempeño de las empresas brasileñas, frente a las empresas mexicanas, o si las empresas de un determinado sector tienen mejores o peores comportamientos que las de otro sector en particular. Estos primeros análisis, que resultan muy sencillos de aplicar, le brindan al auditor la posibilidad de construir o determinar un panorama o perspectiva general acerca de la información que está analizando, información que deberá estar sujeta a posteriores análisis más profundos y detallados.

\section{- Ejemplo II}

Una vez desarrollada la primera parte del ejemplo, supongamos ahora que el auditor decidió utilizar el muestreo aleatorio simple sobre la cartera que tiene una de las empresas de la lista, ya que de acuerdo al juicio del auditor y al tamaño de la población de clientes de esta compañía, no resultaría práctico ni viable analizar todos los datos.

En este sentido, el primer paso para el cálculo de la muestra es determinar su tamaño, es decir, cuántos elementos de una determinada población serán elegidos de forma aleatoria. La respuesta a esta pregunta dependerá, entre otras cuestiones, de los objetivos del estudio, de las características de la población, del tiempo y recursos que se tienen para realizar el trabajo $y$, lógicamente también, de la materialidad o importancia relativa que tengan las partidas analizadas. En nuestro ejemplo, supongamos que el auditor decide que el tamaño de la muestra será de 50 elementos, después de tener en cuenta los diferentes factores comentados anteriormente.

Para determinar la muestra dentro de la población total de clientes de esta compañía que, supongamos, sea también de 250 elementos, el auditor podría recurrir al uso de números aleatorios, los cuales pueden generarse muy fácilmente, con la función "aleatorio" en Excel. Una vez generada esta tabla de números aleatorios, 
será utilizada para la determinación de la muestra, esto puede hacerse de diversas maneras; por ejemplo, sistemáticamente, es decir, cada uno de los 250 clientes tendrá asignado un código que iría desde 001 hasta 250 y se comenzaría a recorrer la tabla desde arriba hacia abajo, leyendo solamente los tres primeros dígitos de cada fila, que corresponderían a algunos de los 250 elementos ya codificados, hasta completar la muestra de 50 .

En la tabla 2 se muestra un extracto de lo que sería esta tabla de números aleatorios y cómo se utiliza para la determinación de la muestra de 50 elementos.

\begin{tabular}{|c|c|c|}
\hline Código & Cliente & Núm. aleatorio \\
\hline 001 & Cliente A & 474065789959690 \\
\hline 002 & Cliente B & 568497500234799 \\
\hline 003 & Cliente C & 453546873183514 \\
\hline 004 & Cliente D & 1235468665575847 \\
\hline 005 & Cliente $\mathrm{E}$ & 025876454684684 \\
\hline 006 & Cliente $\mathrm{F}$ & 089546456456456 \\
\hline 007 & Cliente G & 256876845486456 \\
\hline 008 & Cliente $\mathrm{H}$ & 354678641548453 \\
\hline 009 & Cliente I & 156546456435487 \\
\hline 010 & Cliente J & 013985304687925 \\
\hline 011 & Cliente K & 043578211489635 \\
\hline 012 & Cliente L & / 020669038789509 \\
\hline 013 & Cliente M & 573216131836425 \\
\hline 014 & Cliente N & 078545329213479 \\
\hline 015 & Cliente $\mathrm{O}$ & 027895165735279 \\
\hline 016 & Cliente P & 258671134547894 \\
\hline 017 & Cliente Q & 078954752669878 \\
\hline 018 & Cliente R & 323145879654723 \\
\hline 019 & Cliente S & 人 009829968458698 \\
\hline 020 & Cliente T & 129230376660881 \\
\hline
\end{tabular}

Tabla 2

Tabla de números aleatorios y determinación de la muestra Fuente: elaboración propia

Con la muestra ya disponible, el auditor podrá hacer todas las revisiones que estime convenientes sobre estos elementos, los cuales configuran una muestra representativa del total de la población que está conformada por 250 clientes.
Lógicamente, el auditor puede seleccionar el método de muestreo que considere más relevante y útil para el trabajo que va a ejecutar, es decir, perfectamente puede seleccionar cualquiera de los otros tres métodos disponibles 
— sistemático, estratificado y de racimo-. Por ejemplo, si el auditor decide utilizar el método de racimo, tendrá que separar la población de 250 clientes en grupos y, sobre cada uno de estos grupos, podrá calcular una muestra aleatoria. Los grupos se podrían establecer, por ejemplo, con base en los productos o servicios que se les suministren a estos clientes o de acuerdo a las cifras de ventas existentes.

\section{- Ejemplo III}

El tercer ejemplo hace referencia a la aplicación de la teoría de la probabilidad. Supongamos que nuestra firma de auditoría ficticia está haciendo una evaluación y análisis sobre la probabilidad de subsistencia o quiebra de las 250 empresas clientes de la firma, es decir, se analizará para las empresas en cuestión o para una muestra de ellas, cuál de los dos eventos es probable que suceda en el futuro: subsistencia o quiebra. Estos dos eventos conforman lo que en estadística se denomina espacio muestral y para este caso resultan ser mutuamente excluyentes.

Ahora bien, teniendo planteado nuestro problema, el auditor tendrá que decidir con qué planteamiento conceptual de probabilidad se queda: el clásico, el de frecuencia relativa o el subjetivo.

El planteamiento clásico responde a una simple fórmula que está dada por el cociente entre el número de resultados en los que se presenta un evento y el número total de resultados posibles. Como lo plantean Richard I. Levin y David S. Rubin (2010), este primer planteamiento tiene el problema de que no resulta muy útil cuando nos enfrentamos a procesos decisionales poco previsibles, lo cual, para el caso de nuestro ejemplo, es precisamente el escenario en el que estamos.

El segundo planteamiento, el de frecuencia relativa, hace alusión a análisis de probabilidades en las que no se puede dar rápidamente una respuesta debido, entre otras cuestiones, a la imprevisibilidad de la situación o a la gran cantidad de factores que pueden influir en que uno $\mathrm{u}$ otro resultado se presente. En este segundo planteamiento, la probabilidad se define como "la frecuencia relativa observada de un evento durante un gran número de intentos o la fracción de veces que un evento se presenta a la larga, cuando las condiciones son estables" (Levin \& Rubin, 2010).

En términos más simples, el planteamiento de frecuencia relativa tiene en cuenta los eventos pasados que se hayan presentado; por ejemplo, en los últimos años cuántas empresas se han quebrado y cuántas empresas han subsistido en promedio en un determinado país. Esta información sirve de base para predecir la probabilidad de que cualquiera de estos dos eventos suceda o se presente en el futuro. Esta segunda opción podría ser más útil que la primera para el ejemplo presentado, pero hay que tener especial cuidado en revisar y analizar un número suficiente de resultados pasados.

Finalmente, según lo planteado por Richard I. Levin y David S. Rubin (2010), el planteamiento subjetivo se basa en las creencias de las personas que efectúan la estimación de la probabilidad, considerando la evidencia que tales personas tengan sobre el hecho en particular que se está analizando; este método corresponde a situaciones que no se presentan de forma repetitiva, situaciones específicas, las cuales es- 


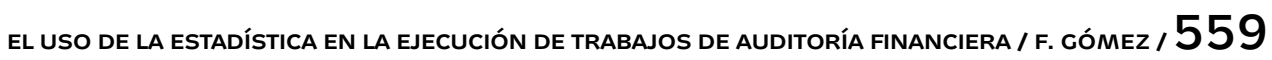

tán muy presentes cuando nos referimos a ciencias sociales o administrativas.

Habiendo revisado muy brevemente estos tres planteamientos conceptuales acerca de la teoría de la probabilidad, para nuestro ejemplo, podríamos desechar rápidamente el primero de ellos y seleccionar el de frecuencia relativa o el subjetivo. Teniendo en cuenta que es probable que haya datos estadísticos previos que nos den luces acerca de la permanencia o no en el tiempo de las empresas de un país, de las compañías de un sector de la economía específico y de las empresas con un tamaño en particular, podríamos concluir que la utilización del planteamiento de frecuencia relativa, tal vez sería lo más acertado, ya que mediante esta aproximación conceptual, podríamos examinar el comportamiento financiero de las organizaciones en cuanto a su subsistencia o quiebra y, a partir de allí, establecer la probabilidad de ocurrencia en el escenario de tiempo previsto. Todos los datos necesarios para llevar a cabo este tipo de análisis, podrían ser extraídos de información directamente recabada de las empresas analizadas o de organismos estatales o privados que recopilan información empresarial, por ejemplo, para el caso de Colombia, la Superintendencia de Industria y Comercio, las cámaras de comercio o la ANDI (Asociación Nacional de Empresarios de Colombia).

La información resultante que tendría en sus manos el auditor podría serle de gran utilidad, ya que si se tiene el conocimiento de la probabilidad de subsistencia o quiebra de un grupo de empresas de un determinado sector de la economía o de un tamaño en particular, el auditor podría centrar y profundizar sus es- fuerzos en los trabajos de auditoría financiera que ejecute en compañías que estén dentro de tales criterios, lo que contribuye a disminuir la probabilidad de quiebra o a maximizar la probabilidad de subsistencia, gracias a las revisiones efectuadas y las recomendaciones realizadas.

\section{- Ejemplo IV}

Finalmente, en el último caso, vamos a suponer que la firma de auditoría de nuestro ejemplo quiere analizar y determinar si es posible establecer un perfil financiero específico tanto para las empresas que han tenido opiniones o dictámenes de auditoría positivos como negativos, es decir, se espera encontrar signos o características financieras que identifiquen a las empresas que conforman cada uno de los dos grupos.

Si los análisis y mediciones demuestran que lo anterior es posible, esta información podría ser de utilidad para la firma de auditoría al realizar evaluaciones futuras en otras empresas, ya que sería posible establecer un sistema de categorización o alerta previa al inicio de un proceso de auditoría, de manera que las revisiones se podrían centrar en ciertas compañías que, según esta clasificación, tendrían un riesgo mayor, sin que esto signifique no evaluar a las demás.

Supongamos nuevamente que nuestra firma de auditoría tiene una base de datos de 250 empresas clientes sobre las cuales ya ha efectuado trabajos de auditoría, y sobre esta población se determina una muestra, la cual estará dividida en dos grupos: las empresas que han tenido dictámenes de auditoría positivos y las que han tenido dictámenes negativos. Para ambos grupos se establecen los promedios en una serie de 
variables financieras previamente determinadas (cifras de ventas, utilidad neta, activos totales y patrimonio neto), las cuales son identificativas de la posición financiera de las organizaciones, de la misma manera como han sido trabajadas este tipo de variables en investigaciones como las de Fabio Gómez y Diego Fernando Católico (2009,
2010) y Pedro Lorca-Fernández y Javier de Andrés-Suárez (2007).

Sobre esta información y siguiendo a Damodar N. Gujarati (1997), quien plantea que uno de los primeros pasos cuando se analizan datos es lo que corresponde a su graficación, tales promedios se presentan en la tabla 3 y el gráfico 1.

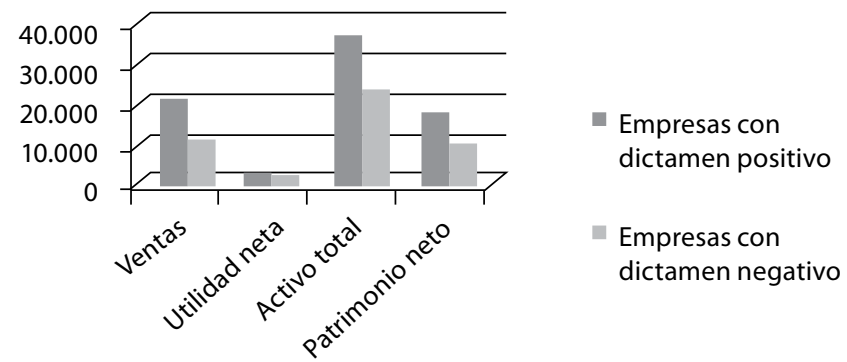

Gráfico 1

Medias de las variables financieras de las empresas con dictámenes positivos y negativos Fuente: elaboración propia

\begin{tabular}{lcc}
\hline & $\begin{array}{c}\text { Empresas } \\
\text { con dictamen } \\
\text { positivo }\end{array}$ & $\begin{array}{c}\text { Empresas } \\
\text { con dictamen } \\
\text { negativo }\end{array}$ \\
\hline Ventas & $19.736,80$ & $9.979,32$ \\
Utilidad neta & $1.360,06$ & 400,89 \\
Activo total & $35.097,41$ & $21.820,14$ \\
Patrimonio & $15.899,29$ & $8.522,97$ \\
neto & & \\
\hline
\end{tabular}

Tabla 3

Medias de las variables financieras de las empresas con dictámenes positivos y negativos

Fuente: elaboración propia

La tabla y el gráfico presentados le permiten a quien analiza la información formarse una idea general respecto al comportamiento promedio de estas cuatro variables en la muestra de empresas analizada, tanto para las empresas que cuentan con dictamen positivo como las que tienen dictamen negativo. Los datos muestran una clara diferencia en las cifras de uno y otro grupo; ahora, los siguientes análisis buscarán verificar si estas diferencias resultan estadísticamente significativas o simplemente son producto del azar, de manera que se pueda determinar si es posible establecer un perfil financiero específico en cada uno de los dos grupos estudiados.

Teniendo claro el análisis que se quiere realizar, inicialmente se procede a la formulación de la hipótesis alterna y de la hipótesis nula, las cuales son las siguientes:

$H_{1}$ : Hay una diferencia estadísticamente significativa entre la media de las variables financieras de las empresas que tienen dictamen positivo de auditoría y la media de las variables financieras de las empresas que tienen dictamen negativo de auditoría. 


\section{EL USO DE LA ESTAdíSTICA EN LA EJECUCIÓN DE TRABAJOS DE AUdITORía FINANCIERA / F. GóMEZ / $\mathbf{5 6 1}$}

$H_{0}$ : No hay una diferencia estadísticamente significativa entre la media de las variables financieras de las empresas que tienen dictamen positivo de auditoría y la media de las variables financieras de las empresas que tienen dictamen negativo de auditoría.

Para establecer si las diferencias que se observan a simple vista son estadísticamente significativas, se llevará a cabo un contraste de hipótesis mediante la utilización de dos técnicas de análisis, $T$ de Student, y test no paramétrico de medias, en cada una de las 4 variables estudiadas para cada uno de los dos grupos, es decir, las que cuentan con dictamen positivo y las que tienen dictamen negativo. Es importante tener en cuenta que previo a los contrastes de hipótesis, es necesario aplicar un examen de normalidad a las variables analizadas, es decir, si las cifras estudiadas en cada una de las cuatro (4) variables financieras objeto de estudio, siguen una distribución normal.

Lo anterior se requiere, debido a que si tales datos no cumplen con el supuesto de normalidad, los resultados de los contrastes de hipótesis - en lo que concierne a la utilización de la primera técnica- no serían confiables y robustos, por lo tanto, no se podrían sacar conclusiones fiables sobre los resultados.

Una vez aplicados los análisis de normalidad, los resultados indican que solo la variable de utilidad neta cumple este patrón, es decir, los datos en esta variable siguen una distribución normal, con lo cual los contrates de hipótesis mediante la técnica de análisis T de Student solo serían confiables para esta variable; por esta razón, se utiliza una segunda técnica de análisis: test no paramétrico de medias, la cual no parte del supuesto de que los datos siguen una distribución normal, lo cual soluciona la problemática planteada y genera resultados más concluyentes, según lo explicado por Javier Gil-Flores $(2005)^{2}$. Una vez hecho este procedimiento previo, se procede a aplicar los contrastes de hipótesis mediante las dos técnicas descritas. Los resultados de este análisis se presentan en la tabla 4.

De acuerdo a los resultados de los diferentes contrastes de hipótesis presentados en la tabla 4, las cifras indican que para el caso de la primera técnica de análisis, no hay diferencias estadísticamente significativas en las variables financieras de las empresas con dictamen positivo respecto a las compañías con dictamen negativo.

Ahora bien, al aplicar la segunda prueba, test no paramétrico de medias, los resultados indican que en ninguna de las cuatro variables se presentan diferencias estadísticamente significativas en las empresas que tienen dictamen positivo, respecto a las que tienen dictamen negativo, con lo cual se puede concluir que no es posible establecer un perfil financiero, al menos no claramente, para futuros procesos de auditoría que puedan servir para construir un sistema de categorización o alerta previa al inicio de este tipo de evaluaciones; se acepta así la hipótesis nula y se rechaza la hipótesis alterna.

2 El tema del contraste de hipótesis puede ser revisado y profundizado en el libro de Richard I. Levin y David S. Rubin (2010), que le dedica dos capítulos. 


\begin{tabular}{|c|c|c|c|c|c|c|}
\hline \multirow[b]{2}{*}{ Variable } & \multirow[b]{2}{*}{ Medida } & \multirow[b]{2}{*}{$\begin{array}{c}\text { Empresas } \\
(n=50)\end{array}$} & \multicolumn{2}{|c|}{$\begin{array}{c}\text { Dictamen } \\
\text { positivo o negativo }\end{array}$} & \multicolumn{2}{|c|}{ Test de medias } \\
\hline & & & $\begin{array}{l}\text { Dictamen } \\
\text { positivo } \\
(\mathrm{n}=25) \\
\end{array}$ & $\begin{array}{c}\text { Dictamen } \\
\text { negativo } \\
(n=25)\end{array}$ & $\begin{array}{l}\text { T de Student } \\
\text { (Significancia) }\end{array}$ & $\begin{array}{c}\text { No } \\
\text { paramétricos } \\
\text { (Significancia) }\end{array}$ \\
\hline \multirow{2}{*}{ Ventas } & Media & $14.858,06$ & $19.736,80$ & $9.979,32$ & \multirow{2}{*}{$(0,209)$} & \multirow{2}{*}{$(0,808)$} \\
\hline & Mediana & $4.652,30$ & $5.779,60$ & $3.666,80$ & & \\
\hline \multirow{2}{*}{$\begin{array}{l}\text { Utilidad } \\
\text { neta }\end{array}$} & Media & 880,48 & $1.360,06$ & 400,89 & \multirow{2}{*}{$(0,071)$} & \multirow{2}{*}{$(0,101)$} \\
\hline & Mediana & 278,45 & 336,60 & 207,40 & & \\
\hline \multirow{2}{*}{ Activo total } & Media & $28.458,78$ & $35.097,41$ & $21.820,14$ & \multirow{2}{*}{$(0,435)$} & \multirow{2}{*}{$(0,101)$} \\
\hline & Mediana & $6.370,90$ & $5.806,30$ & $7.487,10$ & & \\
\hline \multirow{2}{*}{$\begin{array}{l}\text { Patrimonio } \\
\text { neto }\end{array}$} & Media & $12.211,13$ & $15.899,29$ & $8.522,97$ & \multirow{2}{*}{$(0,348)$} & \multirow{2}{*}{$(0,884)$} \\
\hline & Mediana & $3.201,60$ & $3.105,90$ & $3.365,00$ & & \\
\hline
\end{tabular}

Tabla 4

Contrastes de hipótesis de las variables financieras

Fuente: elaboración propia

Es importante aclarar que los resultados obtenidos podrían ser diferentes, si el análisis se hubiera efectuado con razones financieras, es decir, si se hubiera trabajado con ratios de endeudamiento, de liquidez y de utilidad, entre otras, con lo cual se podría establecer un posible perfil financiero, lógicamente, si los resultados de los contrastes de hipótesis así lo demuestran.

La definición de perfiles financieros en las empresas podría fortalecer el trabajo del auditor financiero, por ejemplo, para el análisis de ciertas partidas específicas de los estados financieros o, también, para la evaluación del sistema de control interno de una determinada entidad.

Los cuatro ejemplos descritos anteriormente (agrupación de datos, muestreo, probabilidades y contraste de hipótesis) son apenas una pequeña muestra de lo relevante y útil que este tipo de herramientas puede ser cuando se está analizando información, actividad que al fin de cuentas está presente de forma permanente cuando se realizan trabajos de auditoría financiera.

\section{Conclusiones}

La auditoría es una función de la profesión contable que parece estar en crisis desde hace varios años; esto ha sido evidenciado en diversos trabajos como los de María Antonia García-Benau y Antonio Vico-Martínez (2003) y Fabio Gómez (2014), que analizan este problema en países de tradición latina, como Colombia, México y España, en los que el avance y el desarrollo de la auditoría y sus técnicas están condicionados por diversos factores (políticos, sociales, económicos, etc.). De otra parte, esto no ocurre, al menos no tan marcadamente, en países anglosajones (Estados Unidos, Reino Unido y Australia), donde la estandarización contable y la de la auditoría tienen mejores niveles de formación, metodologías y prácticas.

Esta problemática se está tratando de solucionar desde diferentes ópticas, por ejemplo, con procesos de formación más rigurosos, normalización internacional, leyes más estrictas, 


\section{EL USO DE LA ESTAdístiCA EN LA EJECUCIÓN DE TRABAJOS DE AUDITORÍA FINANCIERA / F. GóMEZ / $\mathbf{5 6 3}$}

masificación en el uso de las TIC en los procesos de auditoría y extensión en la responsabilidad del auditor, por nombrar algunas de las posibles soluciones.

Ahora bien, en este trabajo se quiso contribuir a la solución de esta problemática, desde el punto de vista de los procesos de formación, en lo que concierne al uso de la estadística en la ejecución de trabajos de auditoría financiera; teniendo en cuenta que según los resultados de investigaciones previas, este es un punto de notoria debilidad entre los profesionales contables en Colombia.

Las temáticas desarrolladas en el presente documento pretenden "sembrar" en el profesional contable el interés por el uso de la estadística en los procesos de auditoría financiera, con énfasis en las ventajas y la utilidad que supone su uso.

Resulta claro para el autor de este escrito, que no se puede pretender enseñar estadística o econometría en un artículo, pero sí se pueden sentar las bases o fundamentos que le permitan ver al contador público/auditor los enormes beneficios de usar estas importantes herramientas, no solo en el trabajo como auditor, sino también en el desempeño de sus funciones como contador público, beneficios como el ahorro de tiempo en la ejecución de trabajos de auditoría, un mayor grado de seguridad razonable en las conclusiones de las auditorías realizadas y, en general, la mejora en la calidad del trabajo efectuado.

Sentadas estas bases, ya dependerá de cada profesional subsanar sus propias debilidades mediante el seguimiento de programas de formación y actualización en estas áreas. Este reto no solo les compete a los profesionales en ejercicio o a los futuros contadores públicos; las instituciones universitarias tienen una responsabilidad y juegan un papel fundamental en el objetivo último de mejorar la calidad de sus futuros egresados, lo cual se podrá lograr mediante la inserción de estos temas en sus programas de pregrado y posgrado o en cursos de extensión o diplomados, para dar respuesta a una necesidad manifiesta en cuanto a la formación de los profesionales contables.

Esta tarea que deberán hacer tanto las instituciones universitarias como los contadores públicos, podrá llevarse a cabo con la participación de organismos contables nacionales, que orienten y faciliten este proceso, todo ello con el ánimo de que el contador público/auditor ofrezca un trabajo con elevados niveles de calidad, en beneficio de las organizaciones a las que les presta sus servicios y, lógicamente, en favor de la profesión contable en general.

\section{Referencias}

Alonso-Antón, Aurora; Fernández-Macho, Francisco Javier \& Gallastegui-Zulaica, Inmaculada (2005). Econometría. Madrid: Editorial Pearson Prentice Hall.

Arens, Alvin A. \& Loebbecke, James (1981).

Applications of Statistical Sampling to

Auditing. New Jersey: Editorial Prentice Hall.

Barua, Anitesh; Kriebel, Charles H. \&

Mukhopadhyay, Tridas (1995). Information

Technology and Business Value: An

Analytic and Empirical Investigation.

Information Systems Research, 6 (1), 3-23. 
Benvenuto-Vera, Ángelo (2004). Los delitos informáticos (Ley 19.223 Chile) y la función de auditoría informática. CAPIC Review, Conferencia Académica Permanente de Investigación Contable, 2 , 27-34. Disponible en: http://www.capic. cl/wp-content/uploads/2015/09/TEMA-2BENVENUTO.pdf

Bonsón-Ponte, Enrique (2000). La auditoría de cuentas en la economía digital: hacia un nuevo paradigma. Revista de la Asociación Española de Contabilidad y Administración de Empresas, AECA, 52, 56-59. Disponible en: http://www.aeca1.org/revistaeca/ revista52/52.pdf

Cuéllar-Mejía, Guillermo Adolfo (2009). Clasificación de la Auditoría. En Teoría general de la auditoría y revisoría fiscal, Unidad tres, 1-36. Disponible en: http:// fccea.unicauca.edu.co/old/tgarf/marcos. html

Deschamps, Marco (2007). Muestreo estadístico aplicado a auditoría financiera. Disponible en: http://studylib.es/doc/5683773/ muestreo-estad\%C3\%ADstico-aplicado-aauditor\%C3\%ADa-financiera

Escuder-Vallés, Roberto \& Méndez-Martínez, Salvador (2002). Métodos de muestreo estadístico aplicados a la auditoría. Valencia: Editorial Tirant lo Blanch.

García-Benau, María Antonia \& Vico-Martínez, Antonio (2003). Los escándalos financieros y la auditoría: pérdida y recuperación de la confianza en una profesión en crisis. Revista Valenciana de Economía y Hacienda, 7, 25-48. Disponible en: http://www. academiacarceller.net/apuntes/continternacional/debate2-7.pdf.pdf

Gil-Flores, Javier (2005). Aplicación del método Bootstrap al contraste de hipótesis en la investigación educativa. Revista de Educación, 336, 251-265.

Goldberger, Arthur, S. (1991). A Course in Econometrics. Cambridge, Massachusetts: Harvard University Press.

Gómez, Fabio (2014). Competencia digital en la auditoría. Soporte o carga en el ejercicio profesional de los auditores. Cuadernos de Contabilidad, 15 (37), 135-151. Disponible en: http://cuadernosdecontabilidad. javeriana.edu.co/vol15_n_37/vol15_37_5. pdf

Gómez, Fabio \& Católico, Diego (2009). Revelación y divulgación de la información financiera y no financiera on-line de las 500 empresas más representativas en Colombia. Cuadernos de Contabilidad, 10 (27), 269318. Disponible en: http://revistas. javeriana.edu.co/index.php/cuacont/article/ view/3210/2442

Gómez, Fabio \& Católico, Diego (2010). Relación de la presentación de información de negocios on-line con las variables financieras en las empresas colombianas. Revista Facultad de Ciencias Económicas: Investigación y Reflexión, Universidad Militar Nueva Granada, 18 (1), 205-224. Disponible en: https://revistas.unimilitar.edu.co/index. php/rfce/article/view/2289/2009

Gubba, Hugo; Gutfraind, Jorge; Rodríguez, Rubén \& Villarmarzo, Ricardo (2001). Auditoría de evidencia virtual: cambio filosófico o adaptación del profesional 


\section{EL USO DE LA ESTADístiCA EN LA EJECUCIÓN DE TRABAJOS DE AUDITORÍA FINANCIERA / F. GóMEZ / 565}

a las nuevas modalidades. Revista de Antiguos Alumnos del Instituto de Estudios Empresariales de Montevideo, IEEM, 4 (1), 32-44.

Gujarati, Damodar N. (1992). Essentials of Econometrics. New York: Editorial McGrawHill.

Gujarati, Damodar N. (1997). Econometría básica. $3^{a}$ ed., Bogotá: Editorial McGrawHill Interamericana.

Hernández-Sampieri, Roberto; Fernández, Carlos \& Baptista, Pilar (2010). Metodología de la investigación. $5^{\mathrm{a}}$ ed., México: McGrawHill.

Jokovich, Ghanzov (2013). Statistical Sampling in Auditing. International Journal of Accounting and Financial Management, 16, 892-898. Disponible en: http://universalrg. org/FullText/12013161.pdf

Levin, Richard I. \& Rubin, David S. (2010). Estadística para administración y economía. $7^{\mathrm{a}}$ ed., México: Editorial Pearson.

López-Ruiz, Víctor Raúl \& Nevado-Peña, Domingo (1999). El modelo econométrico: una herramienta al alcance del auditor de cuentas. Documento de Trabajo Serie 2, No. 2, Facultad de Ciencias Económicas y Empresariales de Albacete, Universidad Castilla La Mancha, España.

Lorca-Fernández, Pedro \& Andrés-Suárez, Javier de (2007). Efectos de la implantación de sistemas integrados de gestión (ERP) en las grandes empresas españolas. Revista Española de Financiación y Contabilidad, REFC, 36 (135), 595-623. Disponible en: https://www.researchgate.net/ publication/271821544_Efectos_de_la_ implantacion_de_sistemas_integrados_de_ gestion_ERP_en_las_grandes_empresas_ espanolas

Luo, Wenhong; Cook, David; Joseph, Jimmie \& Ganapathy, Bopana (2000). An Exploratory Framework for Understanding Electronic Bill Presentment and Payment -EBPP - Model Selection. Human Systems Management, 19 (4), 255-264.

Méndez-Martínez, Salvador; EscuderVallés, Roberto \& Mar-Molinero, Cecilio (2001). Una aplicación de los métodos de remuestreo a la auditoría de estados financieros. Revista Estadística Española, 43 (147), 45-61. Disponible en: http://www. ine.es/ss/Satellite?blobcol=urldata\&blobhe ader $=$ application $\% 2 F p d f \& b l o b h e a d e r n a m$ e1=Content-Disposition\&blobheadervalue1 $=$ attachment $\% 3 \mathrm{~B}+$ filename $\% 3 \mathrm{D} 812 \% 2 \mathrm{~F} 9$ 44\%2F147_3.pdf\&blobkey=urldata\&blobta ble $=$ MungoBlobs\&blobwhere $=812 \% 2 \mathrm{~F} 944$ \%2F147_3.pdf\&ssbinary=true

Miró i Martínez, Pau; Debón-Aucejo, Ana \& Crespo-Abril, Fortunato (2006). Métodos estadísticos en economía. Valencia: Editorial Universidad Politécnica de Valencia.

Porter, William Thomas \& Burton, John (1980). Auditoría: un análisis conceptual. México: Editorial Diana.

Rivera, Luz M. (2006). Estadística. Ponce: Universidad Interamericana de Puerto Rico. Disponible en: http://cremc.ponce.inter. edu/topicos/estad.htm.

Rubio-Andrada, Luis (2005). Métodos estadísticos para la administración y dirección de empresas: Supuestos resueltos de contrastes no paramétricos. Madrid: Vision Net. 
Rueda-Delgado, Gabriel; Pinzón-Pinto, Jorge Emiro \& Patiño-Jacinto, Ruth Alejandra (2013). Los currículos de los programas académicos de Contaduría Pública, tras la enseñanza de lo internacional y la globalización en la contabilidad: necesidades de ajuste más allá de respuestas técnicas. Cuadernos de Contabilidad, 14 (35), 639-667. Disponible en: http://revistas. javeriana.edu.co/index.php/cuacont/article/ view/7110/5632

Samuelson, Paul; Koopmans, Tjalling \& Stone, Richard (1954). Report of the Evaluative Committee for Econometrica. Econometrica, 22 (2), 141-146. Disponible en: https:// www.econometricsociety.org/publications/ econometrica/issue/1954/04/

Santos, Gerardo \& Patiño-Jacinto, Ruth Alejandra (2009). Planes de Estudio de Contaduría Pública en Colombia y las propuestas de formación profesional. Revista Internacional Legis de Contabilidad y Auditoría, 37, 131-163.

Stringer, Kenneth (1963). Practical Aspects of Statistical Sampling in Auditing. Proceedings of the Business and Economic Statistics Section (American Statistical Association), 405-411. Valderrama-Prieto, Marcos Ancisar (2002). ERP y lo contable. Working Paper, Pontificia Universidad Javeriana, Departamento de Ciencias Contables, Bogotá. Disponible en: http://entidadpublica.co/wp-content/ uploads/2013/05/erp_fundamental.pdf Wooldridge, Jeffrey (2007). Introducción a la econometría - Un enfoque moderno. $2^{\mathrm{a}} \mathrm{ed}$., Madrid: Ediciones Paraninfo.

- Fecha de recepción: 29 de enero de 2016

- Fecha de aceptación: 14 de mayo de 2016

- Disponible en línea: 14 de diciembre de 2016

\section{Para citar este artículo}

Fabio Enrique Gómez Menesesr(2016). El uso de la estadística en la ejecución de trabajos de auditoría financiera. Cuadernos de Contabilidad, 17 (44), 545-573. https://doi.org/10.11144/Javeriana.cc1744.ueet 
EL USO DE LA ESTAdíSTICA EN LA EJECUCIÓN DE TRABAJOS DE AUDITORÍA FINANCIERA / F. GóMEZ / $\mathbf{5 6 7}$

\section{Anexo 1}

Datos empresariales cuantitativos utilizados

\begin{tabular}{|c|c|c|c|c|c|c|}
\hline \# & Empresa & País & $\begin{array}{c}\text { Ventas } \\
2012 \text { US\$ } \\
\text { millones }\end{array}$ & $\begin{array}{c}\text { Utilidad neta } \\
2012 \text { US\$ } \\
\text { millones }\end{array}$ & $\begin{array}{c}\text { Activo total } \\
2012 \text { US } \$ \\
\text { millones }\end{array}$ & $\begin{array}{c}\text { Patrimonio } \\
\text { neto } 2012 \\
\text { US } \$ \text { millones }\end{array}$ \\
\hline 1 & Empresa A & $\mathrm{Bra}$ & $137.694,9$ & $10.365,8$ & $331.644,9$ & $167.888,3$ \\
\hline 2 & Empresa B & Ven & $124.459,0$ & $4.237,0$ & $218.424,0$ & $75.828,0$ \\
\hline 3 & Empresa C & Méx & $59.778,0$ & $7.052,5$ & $77.426,6$ & $23.276,5$ \\
\hline 4 & Empresa D & Bra & $45.770,5$ & $4.763,2$ & $130.619,8$ & $74.572,2$ \\
\hline 5 & Empresa E & $\mathrm{Bra}$ & $39.210,7$ & 925,4 & $8.922,4$ & $5.069,2$ \\
\hline 6 & Empresa F & $\mathrm{Col}$ & $37.735,2$ & $8.451,9$ & $56.813,8$ & $36.837,2$ \\
\hline 7 & Empresa G & $\mathrm{Bra}$ & $37.042,7$ & 614,4 & $24.348,5$ & $10.085,9$ \\
\hline 8 & Empresa H & Méx & $32.242,6$ & $1.795,1$ & $17.144,0$ & $10.774,6$ \\
\hline 9 & Empresa I & $\mathrm{Bra}$ & $26.385,8$ & 494,7 & $7.487,1$ & $2.931,4$ \\
\hline 10 & Empresa J & Bra & $25.446,8$ & 487,6 & $15.579,9$ & $8.482,7$ \\
\hline 11 & Empresa K & Bra & $24.920,2$ & 514,4 & $17.321,4$ & $4.156,9$ \\
\hline 12 & Empresa L & Bra & $22.875,3$ & 379,7 & $4.372,2$ & $1.194,6$ \\
\hline 13 & Empresa M & Chi & $22.770,0$ & 232,5 & $22.622,0$ & $6.442,4$ \\
\hline 14 & Empresa N & Chi & $19.116,3$ & 564,1 & $20.213,1$ & $7.128,2$ \\
\hline 15 & Empresa O & Bra & $18.812,5$ & 8,1 & $8.260,8$ & $3.876,1$ \\
\hline 16 & Empresa P & Bra & $18.586,6$ & 697,6 & $25.981,5$ & $13.332,8$ \\
\hline 17 & Empresa Q & Méx & $18.379,8$ & $1.597,0$ & $22.824,8$ & $11.974,5$ \\
\hline 18 & Empresa R & Chi & $15.860,4$ & $3.875,3$ & $31.645,0$ & $12.177,8$ \\
\hline 19 & Empresa S & Bra & $15.772,5$ & $5.142,2$ & $26.503,4$ & $14.124,7$ \\
\hline 20 & Empresa T & Ecu & $15.616,5$ & $5.864,5$ & $9.479,9$ & $4.171,4$ \\
\hline 21 & Empresa U & Méx & $15.438,1$ & 693,7 & $11.866,5$ & $4.013,8$ \\
\hline 22 & Empresa V & Bra & $14.688,8$ & 424,9 & $16.540,3$ & $6.555,3$ \\
\hline 23 & Empresa W & Bra & $14.050,9$ & 457,2 & $12.052,8$ & $2.889,7$ \\
\hline 24 & Empresa X & $\mathrm{Bra}$ & $13.955,2$ & 398,0 & $15.058,6$ & $7.114,5$ \\
\hline 25 & Empresa Y & $\operatorname{Arg}$ & $13.639,4$ & 792,3 & $16.233,3$ & $6.347,2$ \\
\hline 26 & Empresa Z & Méx & $13.353,5$ & 156,5 & $10.577,1$ & $3.450,3$ \\
\hline 27 & Empresa AA & Chi & $13.080,5$ & 788,4 & $27.826,6$ & $8.135,8$ \\
\hline 28 & Empresa $\mathrm{AB}$ & Bra & $12.316,7$ & 409,8 & $38.067,7$ & $3.873,7$ \\
\hline 29 & Empresa AC & Bra & $12.132,1$ & 42,6 & $25.577,1$ & $13.597,3$ \\
\hline 30 & Empresa AD & Méx & $12.008,9$ & 890,2 & $16.020,8$ & $5.639,4$ \\
\hline 31 & Empresa AE & Bra & $11.835,8$ & 199,3 & $4.486,2$ & $1.079,6$ \\
\hline 32 & Empresa AF & Bra & $11.720,8$ & 590,1 & $8.228,8$ & $1.014,6$ \\
\hline 33 & Empresa AG & Chi & $11.474,0$ & 775,3 & $18.051,0$ & $6.028,6$ \\
\hline 34 & Empresa AH & Méx & $11.394,5$ & $1.028,3$ & $12.810,9$ & $7.839,8$ \\
\hline 35 & Empresa AI & Bra & $11.180,0$ & 156,9 & $5.912,6$ & $1.445,9$ \\
\hline
\end{tabular}




\begin{tabular}{|c|c|c|c|c|c|c|}
\hline \# & Empresa & País & $\begin{array}{c}\text { Ventas } \\
2012 \text { US\$ } \\
\text { millones }\end{array}$ & $\begin{array}{c}\text { Utilidad neta } \\
2012 \text { US\$ } \\
\text { millones }\end{array}$ & $\begin{array}{c}\text { Activo total } \\
2012 \text { US\$ } \\
\text { millones }\end{array}$ & $\begin{array}{c}\text { Patrimonio } \\
\text { neto } 2012 \\
\text { US } \$ \text { millones }\end{array}$ \\
\hline 36 & Empresa AJ & Bra & $11.133,4$ & $2.042,6$ & $11.324,6$ & $4.913,4$ \\
\hline 37 & Empresa AK & Arg & $10.834,0$ & $1.699,0$ & $15.963,9$ & $11.388,0$ \\
\hline 38 & Empresa AL & Méx & $10.634,9$ & 139,0 & $16.831,4$ & $7.177,1$ \\
\hline 39 & Empresa AM & Méx & $10.182,9$ & $2.364,0$ & $18.785,0$ & $8.305,4$ \\
\hline 40 & Empresa AN & Chi & $9.722,2$ & 11,0 & $20.593,3$ & $5.142,1$ \\
\hline 41 & Empresa AO & Bra & $9.090,7$ & 389,6 & $14.107,2$ & $5.416,1$ \\
\hline 42 & Empresa AP & Bra & $9.182,3$ & 709,0 & $12.776,6$ & $6.769,2$ \\
\hline 43 & Empresa AQ & Bra & $9.033,7$ & $2.090,4$ & $19.952,5$ & $5.893,8$ \\
\hline 44 & Empresa AR & Méx & $8.181,4$ & 908,3 & $12.336,8$ & $3.716,2$ \\
\hline 45 & Empresa AS & Méx & $8.068,2$ & 274,4 & $5.736,4$ & $3.105,9$ \\
\hline 46 & Empresa AT & Méx & $7.658,4$ & 952,0 & $9.617,3$ & $6.122,4$ \\
\hline 47 & Empresa AU & Méx & $7.540,7$ & 760,3 & $6.370,9$ & $3.201,6$ \\
\hline 48 & Empresa AV & Méx & $7.385,4$ & 336,6 & $4.738,3$ & $2.277,2$ \\
\hline 49 & Empresa AW & Bra & $7.367,3$ & 599,9 & $15.207,1$ & $3.374,9$ \\
\hline 50 & Empresa AX & Bra & $7.214,0$ & 42,2 & $2.102,0$ & 148,4 \\
\hline 51 & Empresa AY & $\mathrm{Col}$ & $7.048,5$ & 819,5 & $19.755,6$ & $7.393,8$ \\
\hline 52 & Empresa AZ & Bra & $6.841,4$ & 197,0 & $14.913,1$ & $3.173,4$ \\
\hline 53 & Empresa BA & Bra & $6.836,5$ & 510,9 & $5.116,0$ & $2.024,4$ \\
\hline 54 & Empresa BB & Chi & $6.740,1$ & $1.733,6$ & $12.869,9$ & $8.804,8$ \\
\hline 55 & Empresa BC & Méx & $6.492,4$ & 589,3 & $5.928,1$ & $2.986,1$ \\
\hline 56 & Empresa BD & Bra & $6.219,8$ & $1.442,7$ & $8.340,3$ & $3.857,5$ \\
\hline 57 & Empresa BE & Méx & $6.098,1$ & 402,6 & $11.703,8$ & $4.263,2$ \\
\hline 58 & Empresa BF & Chi & $6.044,3$ & 241,6 & $4.424,0$ & $1.506,2$ \\
\hline 59 & Empresa BG & Bra & $5.971,0$ & 341,5 & $9.490,4$ & $3.258,5$ \\
\hline 60 & Empresa BH & Bra & $5.779,6$ & 624,5 & $11.428,6$ & $5.649,5$ \\
\hline 61 & Empresa BI & Col & $5.775,3$ & 72,2 & $1.986,2$ & 917,8 \\
\hline 62 & Empresa BJ & Méx & $5.563,2$ & 106,1 & $10.282,9$ & $2.598,5$ \\
\hline 63 & Empresa BK & Bra & $5.546,4$ & 200,7 & $5.422,2$ & 396,1 \\
\hline 64 & Empresa BL & Méx & $5.344,1$ & 675,7 & $12.730,9$ & $4.667,2$ \\
\hline 65 & Empresa BM & Méx & $5.271,8$ & 751,5 & $4.955,1$ & $2.845,8$ \\
\hline 66 & Empresa BN & Méx & $5.269,0$ & 418,1 & $5.121,3$ & $2.838,9$ \\
\hline 67 & Empresa BO & Bra & $5.262,8$ & 935,6 & $13.054,0$ & $5.733,1$ \\
\hline 68 & Empresa BP & Per & $5.247,9$ & 25,9 & $2.448,8$ & $1.081,3$ \\
\hline 69 & Empresa BQ & Col & $5.173,4$ & 268,3 & $5.662,7$ & $4.287,4$ \\
\hline 70 & Empresa BR & Méx & $5.109,3$ & 555,1 & $6.564,2$ & $3.819,9$ \\
\hline 71 & Empresa BS & Méx & $4.960,5$ & 86,0 & $3.814,7$ & 871,7 \\
\hline 72 & Empresa BT & Méx & $4.931,8$ & 115,8 & $3.346,5$ & $1.564,3$ \\
\hline 73 & Empresa BU & Méx & $4.889,6$ & 365,2 & $7.719,9$ & $3.249,3$ \\
\hline
\end{tabular}


EL USO DE LA ESTAdíSTICA EN LA EJECUCIÓN DE TRABAJOS DE AUDITORÍA FINANCIERA / F. GóMEZ / 569

\begin{tabular}{|c|c|c|c|c|c|c|}
\hline$\#$ & Empresa & País & $\begin{array}{c}\text { Ventas } \\
2012 \text { US\$ } \\
\text { millones }\end{array}$ & $\begin{array}{c}\text { Utilidad neta } \\
2012 \text { US\$ } \\
\text { millones }\end{array}$ & $\begin{array}{c}\text { Activo total } \\
2012 \text { US } \$ \\
\text { millones }\end{array}$ & $\begin{array}{c}\text { Patrimonio } \\
\text { neto } 2012 \\
\text { US } \$ \text { millones }\end{array}$ \\
\hline 74 & Empresa BV & Bra & $4.873,6$ & 52,8 & $5.137,9$ & $1.750,4$ \\
\hline 75 & Empresa BW & Chi & $4.841,0$ & 182,9 & $8.115,9$ & $2.426,8$ \\
\hline 76 & Empresa BX & Chi & $4.809,5$ & 489,6 & $13.557,6$ & $5.309,7$ \\
\hline 77 & Empresa BY & Bra & $4.802,3$ & 215,8 & $2.881,4$ & 808,5 \\
\hline 78 & Empresa BZ & Chi & $4.759,3$ & 201,7 & $14.046,1$ & $7.980,3$ \\
\hline 79 & Empresa CA & Per & $4.682,0$ & 29,5 & $1.294,4$ & 482,5 \\
\hline 80 & Empresa CB & Bra & $4.639,9$ & 802,8 & $7.891,4$ & $2.401,7$ \\
\hline 81 & Empresa CC & Bra & $4.552,9$ & 433,2 & $5.026,0$ & $2.882,2$ \\
\hline 82 & Empresa CD & Arg & $4.506,8$ & 545,2 & $3.616,0$ & $2.022,1$ \\
\hline 83 & Empresa CE & $\mathrm{Col}$ & $4.431,1$ & 793,5 & $3.834,7$ & $2.276,6$ \\
\hline 84 & Empresa CF & Bra & $4.380,9$ & 14,6 & 526,1 & 67,5 \\
\hline 85 & Empresa CG & Méx & $4.339,8$ & 389,1 & $4.970,8$ & $2.977,5$ \\
\hline 86 & Empresa CH & $\mathrm{Col}$ & $4.338,6$ & $1.335,0$ & $6.101,6$ & $3.456,6$ \\
\hline 87 & Empresa CI & $\mathrm{Col}$ & $4.283,3$ & 196,9 & $4.462,8$ & $1.165,0$ \\
\hline 88 & Empresa CJ & Chi & $4.280,3$ & 140,5 & $13.551,2$ & $6.965,8$ \\
\hline 89 & Empresa CK & $\mathrm{Bra}$ & $4.175,3$ & 342,9 & $10.380,0$ & $5.986,3$ \\
\hline 90 & Empresa CL & Bra & $4.138,9$ & 296,7 & $2.920,7$ & 993,0 \\
\hline 91 & Empresa CM & Bra & $4.029,2$ & 354,9 & $6.502,7$ & $4.247,3$ \\
\hline 92 & Empresa CN & Méx & $3.983,6$ & 427,7 & $9.252,7$ & $2.461,8$ \\
\hline 93 & Empresa CO & Méx & $3.946,3$ & 61,2 & $3.796,3$ & $1.145,5$ \\
\hline 94 & Empresa CP & $\mathrm{Bra}$ & $3.885,1$ & 192,7 & $4.775,7$ & $2.242,0$ \\
\hline 95 & Empresa CQ & $\mathrm{Br} / \mathrm{Py}$ & $3.797,9$ & 520,6 & $18.410,9$ & 100,0 \\
\hline 96 & Empresa CR & $\mathrm{Col}$ & $3.741,4$ & 192,6 & $15.007,9$ & $9.884,8$ \\
\hline 97 & Empresa CS & Bra & $3.725,5$ & 207,4 & $5.738,5$ & $1.480,6$ \\
\hline 98 & Empresa CT & Méx & $3.666,8$ & 87,1 & $7.629,8$ & $1.296,6$ \\
\hline 99 & Empresa CU & Bra & $3.662,9$ & 719,7 & $6.324,3$ & $3.398,7$ \\
\hline 100 & Empresa CV & Chi & $3.553,7$ & $1.615,9$ & $3.699,4$ & $2.420,9$ \\
\hline 101 & Empresa CW & Méx & $3.522,1$ & 513,4 & $3.216,9$ & $1.986,5$ \\
\hline 102 & Empresa CX & $\operatorname{Arg}$ & $3.493,9$ & 6,1 & $1.476,8$ & 268,2 \\
\hline 103 & Empresa CY & Méx & $3.492,6$ & 282,0 & $2.351,3$ & 783,4 \\
\hline 104 & Empresa CZ & Chi & $3.455,7$ & 291,8 & $54.584,2$ & $3.956,8$ \\
\hline 105 & Empresa DA & Bra & $3.440,4$ & 227,3 & $7.194,2$ & 531,8 \\
\hline 106 & Empresa DB & Bra & $3.439,8$ & 123,0 & $2.663,1$ & 917,6 \\
\hline 107 & Empresa DC & Col & $3.376,8$ & 25,3 & 651,3 & 242,0 \\
\hline 108 & Empresa DE & Chi & $3.309,5$ & 174,3 & $1.499,5$ & 571,5 \\
\hline 109 & Empresa DF & Bra & $3.292,0$ & 221,1 & $2.190,1$ & $1.188,4$ \\
\hline 110 & Empresa DG & Bra & $3.213,7$ & 167,2 & $7.061,3$ & $2.184,2$ \\
\hline 111 & Empresa DH & Bra & $3.205,1$ & $1.295,0$ & $5.383,4$ & $1.602,2$ \\
\hline
\end{tabular}




\begin{tabular}{|c|c|c|c|c|c|c|}
\hline$\#$ & Empresa & País & $\begin{array}{c}\text { Ventas } \\
2012 \text { US\$ } \\
\text { millones }\end{array}$ & $\begin{array}{c}\text { Utilidad neta } \\
2012 \text { US\$ } \\
\text { millones }\end{array}$ & $\begin{array}{c}\text { Activo total } \\
2012 \text { US } \$ \\
\text { millones }\end{array}$ & $\begin{array}{c}\text { Patrimonio } \\
\text { neto } 2012 \\
\text { US\$ millones }\end{array}$ \\
\hline 112 & Empresa DI & Per & $3.192,0$ & 314,2 & $4.259,7$ & $1.802,9$ \\
\hline 113 & Empresa DJ & Bra & $3.189,6$ & 225,2 & $3.276,9$ & 382,1 \\
\hline 114 & Empresa DK & Chi & $3.186,3$ & 928,9 & $6.567,4$ & $4.438,2$ \\
\hline 115 & Empresa DL & Bra & $3.182,8$ & 92,7 & $1.371,3$ & 443,0 \\
\hline 116 & Empresa DM & Méx & $3.131,2$ & 47,4 & $1.420,3$ & 731,5 \\
\hline 117 & Empresa DN & Bra & $3.105,3$ & 421,4 & $2.630,5$ & 639,1 \\
\hline 118 & Empresa DO & C.Ri & $3.096,1$ & 2,6 & $1.003,1$ & 660,6 \\
\hline 119 & Empresa DP & Méx & $3.051,8$ & 102,0 & $2.173,5$ & 560,6 \\
\hline 120 & Empresa DQ & Bra & $3.039,4$ & 902,9 & $16.048,9$ & $4.990,5$ \\
\hline 121 & Empresa DR & Méx & $3.036,3$ & 168,5 & $2.162,6$ & $1.468,8$ \\
\hline 122 & Empresa DS & Méx & $3.026,8$ & 253,3 & $2.396,3$ & $1.169,6$ \\
\hline 123 & Empresa DT & Bra & $3.021,2$ & 321,0 & $4.343,3$ & $1.987,0$ \\
\hline 124 & Empresa DU & Bra & $3.016,7$ & 24,4 & 980,2 & 263,1 \\
\hline 125 & Empresa DV & Bra & $3.000,3$ & 803,2 & $2.997,2$ & $1.157,6$ \\
\hline 126 & Empresa DW & Chi & $2.988,1$ & 349,5 & $3.542,1$ & $1.700,8$ \\
\hline 127 & Empresa DX & $\mathrm{Col}$ & $2.971,2$ & 193,5 & $5.012,9$ & $4.149,0$ \\
\hline 128 & Empresa DY & Per & $2.952,3$ & 993,6 & 3223.1 & 2855.0 \\
\hline 129 & Empresa DZ & Bra & $2.927,7$ & 94,5 & 3492.1 & 899.8 \\
\hline 130 & Empresa EA & Bra & $2.890,0$ & 347,8 & 3091.3 & 1911.3 \\
\hline 131 & Empresa EB & Bra & $2.856,8$ & 323,0 & 6767.7 & 2477.2 \\
\hline 132 & Empresa EC & Bra & $2.844,9$ & 394,2 & 3478.2 & 1298.6 \\
\hline 133 & Empresa ED & Arg & $2.839,2$ & 78,3 & 1804.6 & 679.8 \\
\hline 134 & Empresa EE & Per & $2.836,8$ & 379,5 & 2603.2 & 2178.8 \\
\hline 135 & Empresa EF & Bra & $2.822,5$ & 576,1 & 7000.6 & 1619.2 \\
\hline 136 & Empresa EG & Bra & $2.788,6$ & 32,7 & 2831.2 & 446.8 \\
\hline 137 & Empresa EH & Chi & $2.786,4$ & 141,5 & 3649.9 & 1175.6 \\
\hline 138 & Empresa EI & Bra & $2.689,5$ & 267,2 & 2471.9 & 1027.8 \\
\hline 139 & Empresa EJ & Bra & $2.681,0$ & 85,7 & 2210.9 & 857.5 \\
\hline 140 & Empresa EK & Col & $2.669,1$ & 347,6 & 3220.2 & 2776.5 \\
\hline 141 & Empresa EL & Per & $2.660,8$ & 156,2 & 2440.5 & 947.4 \\
\hline 142 & Empresa EM & Bra & $2.655,9$ & $1.132,7$ & 4911.8 & 1114.3 \\
\hline 143 & Empresa EN & Bra & $2.633,1$ & 51,3 & 1634.5 & 1108.2 \\
\hline 144 & Empresa EO & Arg & $2.591,9$ & 124,9 & 3826.8 & 2043.5 \\
\hline 145 & Empresa EP & Bra & $2.583,6$ & 179,4 & 2920.5 & 1104.6 \\
\hline 146 & Empresa EQ & Bra & $2.524,1$ & 55,0 & 4134.0 & 2185.3 \\
\hline 147 & Empresa ER & Méx & $2.512,4$ & 54,0 & 2037.4 & 621.2 \\
\hline 148 & Empresa ES & Arg & $2.511,0$ & 282,5 & 6691.0 & 3365.0 \\
\hline 149 & Empresa ET & Arg & $2.503,4$ & 109,6 & 3538.2 & 2402.5 \\
\hline
\end{tabular}


EL USO DE LA ESTAdíSTICA EN LA EJECUCIÓN DE TRABAJOS DE AUDITORÍA FINANCIERA / F. GóMEZ / $\mathbf{5 7 1}$

\begin{tabular}{|c|c|c|c|c|c|c|}
\hline \# & Empresa & País & $\begin{array}{c}\text { Ventas } \\
2012 \text { US\$ } \\
\text { millones }\end{array}$ & $\begin{array}{c}\text { Utilidad neta } \\
2012 \text { US\$ } \\
\text { millones }\end{array}$ & $\begin{array}{c}\text { Activo total } \\
2012 \text { US\$ } \\
\text { millones }\end{array}$ & $\begin{array}{c}\text { Patrimonio } \\
\text { neto } 2012 \\
\text { US } \$ \text { millones }\end{array}$ \\
\hline 150 & Empresa EU & Bra & $2.486,9$ & 487,4 & 13679.0 & 3480.3 \\
\hline 151 & Empresa EV & Méx & $2.484,7$ & 138,7 & 3011.6 & 1992.3 \\
\hline 152 & Empresa EW & Chi & $2.470,0$ & 230,8 & 5166.6 & 1837.8 \\
\hline 153 & Empresa EX & Méx & $2.456,5$ & 49,9 & 2269.2 & 1216.6 \\
\hline 154 & Empresa EY & Chi & $2.449,4$ & 183,1 & 3217.4 & 1826.5 \\
\hline 155 & Empresa EZ & Chi & $2.429,2$ & 649,2 & 4416.4 & 2132.8 \\
\hline 156 & Empresa FA & Chi & $2.411,2$ & 77,3 & 3782.3 & 1579.3 \\
\hline 157 & Empresa FB & $\mathrm{Col}$ & $2.409,7$ & 152,8 & 14431.8 & 3421.9 \\
\hline 158 & Empresa FC & $\mathrm{Bra}$ & $2.404,0$ & 733,8 & 6001.7 & 2672.0 \\
\hline 159 & Empresa FD & Chi & $2.397,9$ & 264,5 & 1987.0 & 782.8 \\
\hline 160 & Empresa FE & Pan & $2.358,1$ & $1.230,9$ & $8.250,3$ & $6.569,4$ \\
\hline 161 & Empresa FF & $\mathrm{Col}$ & $2.346,4$ & 124,7 & 922,9 & 278,8 \\
\hline 162 & Empresa FG & $\mathrm{Bra}$ & $2.340,5$ & 97,8 & $2.111,4$ & $1.162,6$ \\
\hline 163 & Empresa FH & Chi & $2.327,7$ & 202,9 & $5.831,4$ & $2.477,7$ \\
\hline 164 & Empresa FI & $\operatorname{Arg}$ & $2.298,3$ & 97,9 & $2.437,3$ & 830,5 \\
\hline 165 & Empresa FJ & Chi & $2.277,3$ & 494,9 & $5.806,3$ & $4.487,5$ \\
\hline 166 & Empresa FK & Méx & $2.277,1$ & 159,6 & $2.503,3$ & $1.836,8$ \\
\hline 167 & Empresa FL & Méx & $2.258,9$ & 319,7 & $2.162,3$ & 665,3 \\
\hline 168 & Empresa FM & Per & $2.252,0$ & 626,6 & $4.541,5$ & $3.570,6$ \\
\hline 169 & Empresa FN & Méx & $2.247,6$ & 30,0 & $1.660,8$ & 498,3 \\
\hline 170 & Empresa FO & Chi & $2.247,6$ & 239,1 & $2.771,5$ & $1.281,3$ \\
\hline 171 & Empresa FP & Chi & $2.220,3$ & 48,4 & $2.033,9$ & 588,4 \\
\hline 172 & Empresa FQ & Méx & $2.200,1$ & 121,8 & $3.870,9$ & $1.126,7$ \\
\hline 173 & Empresa FR & Bra & $2.175,1$ & 82,9 & $1.249,3$ & 231,3 \\
\hline 174 & Empresa FS & Pan & $2.163,1$ & 326,5 & $3.479,5$ & $1.536,5$ \\
\hline 175 & Empresa FT & Chi & $2.160,2$ & 116,0 & $3.044,4$ & $1.076,8$ \\
\hline 176 & Empresa FU & Méx & $2.157,4$ & 873,3 & $3.270,9$ & $2.602,1$ \\
\hline 177 & Empresa FV & Bra & $2.140,9$ & 59,9 & $2.408,2$ & $1.338,1$ \\
\hline 178 & Empresa FW & Per & $2.127,0$ & 772,0 & $4.042,7$ & $3.449,7$ \\
\hline 179 & Empresa FX & Bra & $2.104,5$ & 304,1 & $4.107,1$ & $1.570,6$ \\
\hline 180 & Empresa FY & Bra & $2.087,5$ & 258,2 & $5.436,1$ & $1.860,4$ \\
\hline 181 & Empresa FZ & Bra & $2.085,2$ & 83,5 & $2.061,4$ & 362,2 \\
\hline 182 & Empresa GA & $\mathrm{Bra}$ & $2.068,4$ & 225,9 & $1.938,3$ & $1.006,1$ \\
\hline 183 & Empresa GB & Per & $2.060,5$ & 143,6 & $1.978,4$ & 696,4 \\
\hline 184 & Empresa GC & Bra & $2.037,5$ & 368,0 & $6.898,9$ & $2.652,8$ \\
\hline 185 & Empresa GD & Chi & $2.036,2$ & 367,2 & $2.723,5$ & $2.166,1$ \\
\hline 186 & Empresa GE & Méx & $2.034,8$ & 70,1 & 912,9 & 478,3 \\
\hline 187 & Empresa GF & $\mathrm{Bra}$ & $2.033,6$ & 88,5 & $1.182,2$ & 197,6 \\
\hline
\end{tabular}




\begin{tabular}{|c|c|c|c|c|c|c|}
\hline \# & Empresa & País & $\begin{array}{c}\text { Ventas } \\
2012 \text { US } \$ \\
\text { millones }\end{array}$ & $\begin{array}{c}\text { Utilidad neta } \\
2012 \text { US\$ } \\
\text { millones }\end{array}$ & $\begin{array}{c}\text { Activo total } \\
2012 \text { US\$ } \\
\text { millones }\end{array}$ & $\begin{array}{c}\text { Patrimonio } \\
\text { neto } 2012 \\
\text { US\$ millones }\end{array}$ \\
\hline 188 & Empresa GG & Per & $2.032,8$ & 105,4 & $1.140,5$ & 684,5 \\
\hline 189 & Empresa GH & Chi & $2.025,8$ & 210,5 & $2.931,3$ & $1.417,5$ \\
\hline 190 & Empresa GI & Bra & $2.014,2$ & 52,7 & $7.850,8$ & 529,5 \\
\hline 191 & Empresa GJ & Méx & $1.992,5$ & 115,3 & $1.018,3$ & 497,7 \\
\hline 192 & Empresa GK & Méx & $1.985,4$ & 131,0 & $1.598,1$ & $1.097,0$ \\
\hline 193 & Empresa GL & Bra & $1.968,0$ & 38,0 & $2.235,8$ & 454,8 \\
\hline 194 & Empresa GM & $\mathrm{Col}$ & $1.925,5$ & 370,9 & $2.096,0$ & $1.456,9$ \\
\hline 195 & Empresa GN & Bra & $1.895,5$ & 98,8 & $6.193,5$ & $3.361,1$ \\
\hline 196 & Empresa GO & Ecu & $1.893,0$ & 142,4 & $1.327,6$ & 926,0 \\
\hline 197 & Empresa GP & Bra & $1.890,3$ & 711,6 & $2.084,4$ & 749,7 \\
\hline 198 & Empresa GQ & Bra & $1.890,1$ & 173,9 & $1.844,9$ & 638,9 \\
\hline 199 & Empresa GR & Méx & $1.888,4$ & 124,9 & $6.974,8$ & $1.782,9$ \\
\hline 200 & Empresa GS & Chi & $1.878,7$ & 96,4 & $1.372,7$ & 367,3 \\
\hline 201 & Empresa GT & Bra & $1.877,4$ & 791,0 & $7.422,6$ & $6.324,0$ \\
\hline 202 & Empresa GU & Per & $1.876,0$ & 85,6 & $2.377,6$ & $1.137,5$ \\
\hline 203 & Empresa GV & Bra & $1.867,9$ & 146,8 & $1.718,7$ & 636,1 \\
\hline 204 & Empresa GW & Bra & $1.860,9$ & 19,9 & 700,1 & 274,5 \\
\hline 205 & Empresa GX & Per & $1.850,4$ & 283,9 & $1.557,6$ & 527,4 \\
\hline 206 & Empresa GY & Bra & $1.820,4$ & 83,6 & $2.145,8$ & 620,4 \\
\hline 207 & Empresa GZ & Per & $1.816,5$ & 86,4 & $1.600,3$ & 558,1 \\
\hline 208 & Empresa HA & Col & $1.813,4$ & 8,7 & $2.201,5$ & 734,4 \\
\hline 209 & Empresa HB & Bra & $1.810,4$ & 55,5 & $1.676,5$ & 545,8 \\
\hline 210 & Empresa HC & Bra & $1.809,2$ & 76,3 & $1.226,4$ & 867,5 \\
\hline 211 & Empresa HD & Arg & $1.808,9$ & 36,3 & 538,2 & 209,0 \\
\hline 212 & Empresa HE & Bra & $1.806,2$ & 241,4 & $2.568,9$ & $1.014,6$ \\
\hline 213 & Empresa HF & Chi & $1.784,2$ & 29,6 & $1.258,9$ & 659,9 \\
\hline 214 & Empresa HG & Méx & $1.782,5$ & 204,5 & $2.705,1$ & 300,4 \\
\hline 215 & Empresa HH & Bra & $1.773,0$ & 145,8 & $2.445,5$ & $1.078,1$ \\
\hline 216 & Empresa HI & Bra & $1.771,0$ & 21,8 & $1.213,5$ & 241,9 \\
\hline 217 & Empresa HJ & Col & $1.759,4$ & 286,2 & $2.998,4$ & $1.747,8$ \\
\hline 218 & Empresa HK & Chi & $1.757,0$ & 42,7 & $2.411,5$ & 783,0 \\
\hline 219 & Empresa HL & Per & $1.754,2$ & 137,8 & $1.680,0$ & 826,7 \\
\hline 220 & Empresa HM & Bra & $1.746,8$ & 175,0 & $2.230,8$ & 947,6 \\
\hline 221 & Empresa HN & Bra & $1.746,3$ & 116,1 & $8.638,3$ & $2.057,1$ \\
\hline 222 & Empresa HO & Bra & $1.735,3$ & 178,9 & $2.075,2$ & $1.236,6$ \\
\hline 223 & Empresa HP & Bra & $1.735,2$ & 7,4 & $1.894,3$ & 763,7 \\
\hline 224 & Empresa HQ & Bra & $1.734,8$ & 230,3 & $1.476,0$ & $1.129,7$ \\
\hline 225 & Empresa HR & Per & $1.730,8$ & 275,6 & $13.803,8$ & $2.294,4$ \\
\hline
\end{tabular}


EL USO DE LA ESTAdíStICA EN LA EJECUCIÓN DE TRABAJOS DE AUDITORÍA FINANCIERA / F. GóMEZ / $\mathbf{5 7}$

\begin{tabular}{|c|c|c|c|c|c|c|}
\hline \# & Empresa & País & $\begin{array}{c}\text { Ventas } \\
2012 \text { US } \$ \\
\text { millones }\end{array}$ & $\begin{array}{c}\text { Utilidad neta } \\
2012 \text { US\$ } \\
\text { millones }\end{array}$ & $\begin{array}{c}\text { Activo total } \\
2012 \text { US } \$ \\
\text { millones }\end{array}$ & $\begin{array}{c}\text { Patrimonio } \\
\text { neto } 2012 \\
\text { US } \$ \text { millones }\end{array}$ \\
\hline 226 & Empresa HS & Chi & $1.728,0$ & 54,6 & $2.180,3$ & $1.158,1$ \\
\hline 227 & Empresa HT & Bra & $1.722,1$ & 235,7 & $4.418,0$ & $2.442,0$ \\
\hline 228 & Empresa HU & Bra & $1.713,7$ & 20,8 & $2.114,7$ & 670,2 \\
\hline 229 & Empresa HV & Chi & $1.709,0$ & 583,7 & $3.846,2$ & $1.453,9$ \\
\hline 230 & Empresa HW & Bra & $1.692,3$ & 25,9 & 277,0 & 143,8 \\
\hline 231 & Empresa HX & $\mathrm{Col}$ & $1.689,7$ & 4,4 & 776,8 & 247,0 \\
\hline 232 & Empresa HY & Bra & $1.686,9$ & 79,8 & $6.266,5$ & $2.285,5$ \\
\hline 233 & Empresa HZ & Per & $1.665,5$ & 63,8 & $1.700,6$ & 877,2 \\
\hline 234 & Empresa IA & Bra & $1.661,1$ & 224,7 & $3.996,7$ & $1.967,2$ \\
\hline 235 & Empresa IB & Bra & $1.641,3$ & 72,4 & $8.265,2$ & $4.834,8$ \\
\hline 236 & Empresa IC & Bra & $1.635,4$ & 223,3 & $2.584,8$ & $1.467,4$ \\
\hline 237 & Empresa ID & $\mathrm{Col}$ & $1.622,4$ & 10,1 & 596,4 & 65,7 \\
\hline 238 & Empresa IE & Bra & $1.614,8$ & 24,2 & 557,8 & 109,9 \\
\hline 239 & Empresa IF & Bra & $1.603,9$ & 15,6 & $1.634,8$ & 206,3 \\
\hline 240 & Empresa IG & Chi & $1.599,0$ & 49,0 & 847,4 & 150,9 \\
\hline 241 & Empresa IH & Bra & $1.594,6$ & 16,4 & $2.508,4$ & $1.681,0$ \\
\hline 242 & Empresa II & Bra & $1.577,9$ & 119,5 & $2.788,3$ & 725,3 \\
\hline 243 & Empresa IJ & Méx & $1.568,9$ & 106,7 & $2.165,4$ & 566,9 \\
\hline 244 & Empresa IK & Bra & $1.568,8$ & 234,9 & $2.802,4$ & $2.086,8$ \\
\hline 245 & Empresa IL & Méx & $1.565,9$ & 78,2 & $1.639,5$ & 884,4 \\
\hline 246 & Empresa IM & Méx & $1.565,4$ & 132,9 & $1.229,8$ & 538,1 \\
\hline 247 & Empresa IN & Per & $1.563,5$ & 742,7 & $4.588,6$ & $3.964,3$ \\
\hline 248 & Empresa IO & Chi & $1.555,8$ & $1.993,6$ & 928,3 & 562,2 \\
\hline 249 & Empresa IP & Bra & $1.551,7$ & 27,3 & $1.164,0$ & 414,0 \\
\hline 250 & Empresa IQ & Bra & $1.549,7$ & 117,9 & $1.929,9$ & 648,3 \\
\hline
\end{tabular}


